\title{
RDUS
}

Revue de DROIT

UNIVERSITÉ DE SHERBROOKE

Titre : $\quad$ LA VALSE-HÉSITATION DES COURS DE JUSTICE EN CE QUI TOUCHE À L'INTERPRÉTATION DES MODALITÉS DE MODIFICATION CONSTITUTIONNELLE AU CANADA

Auteur(s): $\quad$ Benoît PELLETIER

Revue : $\quad$ RDUS, 2017, volume 47, numéro 1

Pages: $\quad$ 57-108

ISSN : $\quad 0317-9656$

Éditeur : $\quad$ Université de Sherbrooke. Faculté de droit.

URI : $\quad$ http://hdl.handle.net/11143/15287

DOl : $\quad$ https://doi.org/10.17118/11143/15287 
Page vide laissée intentionnellement. 


\section{LA VALSE-HÉSITATION DES COURS DE JUSTICE EN CE QUI TOUCHE À L'INTERPRÉTATION DES MODALITÉS DE MODIFICATION CONSTITUTIONNELLE AU CANADA}

par Benoît PELLETIER*

Depuis de nombreuses années, les tribunaux rendent des décisions dans lesquelles ils se prononcent sur la portée des différentes modalités de modification constitutionnelle applicables à toute époque pertinente. Ils semblent toutefois tergiverser entre une approche purement technique et une approche plutôt téléologique de ces mêmes modalités. Le présent article a essentiellement pour objet de décortiquer toute cette jurisprudence et de mettre en relief la valse-hésitation des tribunaux en ce qui a trait à l'interprétation et à l'application des modalités de modification constitutionnelle au Canada.

Over the past several years, the courts have handed down decisions relating to the impact of various approaches to constitutional change. However, these same tribunals have tended to vacillate between a purely technical approach and one which is more teleological in nature. This article aims at analysing the pertinent jurisprudence while highlighting the ambivalent attitude of the courts when interpreting and applying procedures for amending the Canadian Constitution.

* $\quad$ Professeur titulaire à la Faculté de droit de 1'Université d'Ottawa. L'auteur tient à remercier mesdames Marie-Pierre Boudreau et Émie Dubuc pour leur assistance. Dans le présent article, les références seront omises lorsqu'il y aura des citations. 


\section{SOMMAIRE}

A) Le Renvoi relatif à The Initiative and Referendum Act... 61

B) L'arrêt Blaikie 66

C) L'arrêt Forest. 69

D) Le Renvoi relatif à la Chambre haute.........................70

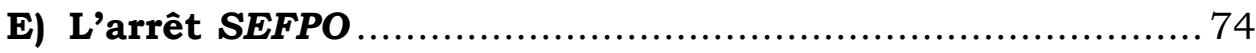

F) L'arrêt N.B. Broadcasting ................................. 76

G) L'arrêt Colombie-Britannique c. Canada ......................79

H) Le Renvoi relatif aux juges de la Cour provinciale .........8 80

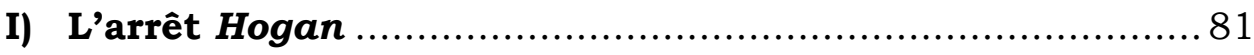

J) Le Renvoi relatif à la sécession du Québec..................88

K) L'arrêt Potter.............................................. 91

L) Le Renvoi relatif à la Loi sur la Cour suprême..............94

M) Le Renvoi relatif à la réforme du Sénat ....................97

CONCLUSION ................................................... 105 


\section{INTRODUCTION}

La modification constitutionnelle au Canada est, depuis longtemps, au cœur de décisions judiciaires fort importantes. La présente étude a essentiellement pour objet d'explorer ces décisions en vue de jeter un éclairage inédit sur les modalités prévues dans la partie V de la Loi constitutionnelle de 1982 (ci-après "Loi de 1982 ")1. De fait, depuis le "rapatriement ${ }^{2}$ " de la Constitution canadienne, la procédure de modification constitutionnelle se trouve contenue dans la partie $\mathrm{V}$ en question et se déploie en différentes modalités, soit la procédure de l'unanimité (art. 41), la procédure $7 / 50$ (art. 38 (1) et 42), la procédure sélective (art. 43), la procédure unilatérale fédérale (art. 44) et la procédure unilatérale provinciale (art. 45).

Comme on s'en doute, la procédure de modification constitutionnelle de la partie V de la Loi de 1982 obéit à une logique vraiment particulière. Toutefois, si l'existence de cette procédure est une chose, l'interprétation qu'en font les tribunaux en est une autre. Or, ces derniers semblent tergiverser entre une approche purement technique et une approche plutôt téléologique ${ }^{3}$ de la procédure en question.

1. Loi constitutionnelle de 1982, constituant l'annexe B de la Loi de 1982 sur le Canada (R.-U.), 1982, c. 11. Elle est entrée en vigueur le 17 avril 1982 (art. 58; TR/82-97).

2. Le " rapatriement " de la Constitution canadienne a été effectué en 1982, en vertu de la Loi de 1982 sur le Canada, préc., note 1. Le mot " rapatriement " est ici mis entre guillemets, car il est plus ou moins exact. En effet, il désigne le fait de rapporter quelque chose (ou de ramener quelqu'un) dans son pays d'origine. Or, le Canada ne possédait pas le pouvoir de modifier sa propre constitution avant que celle-ci soit " rapatriée ".

3. L'interprétation dite téléologique repose sur l'idée voulant que les lois et les textes constitutionnels soient interprétés en fonction des fins recherchées par le législateur ou le constituant. S'il applique cette méthode, l'interprète doit procéder en deux étapes : déterminer le but, l'objet, la finalité de la disposition visée; interpréter le libellé de la disposition en question de la façon qui lui permette de se rapprocher le plus possible de son but, de son objet, de sa finalité. Mélanie SAmson, "Interprétation large et libérale et interprétation contextuelle : conver- 
Quelques décisions illustrent particulièrement bien cette valse-hésitation. Parmi ces décisions, nous avons choisi d'examiner ici celles qui ont été rendues à l'occasion des affaires suivantes :

- In re The Initiative and Referendum Act (ci-après "Renvoi relatif à The Initiative and Referendum Act ")

- Proc. Gen. du Québec c. Blaikie et autres (ci-après "arrêt Blaikie ") 5 ;

- Procureur général du Manitoba c. Forest (ci-après "arrêt Forest ")

- Renvoi : Compétence du Parlement relativement à la Chambre haute (ci-après " Renvoi relatif à la Chambre haute ");

- Le procureur général de l'Ontario c. SEFPO (ci-après " arrêt SEFPO ॥8;

- New Brunswick Broadcasting Co c. Nouvelle-Écosse (Président de l'Assemblée législative) (ci-après "arrêt N.B. Broadcasting "19;

- Colombie-Britannique (Procureur général) c. Canada (Procureur général); Acte concernant le chemin de fer de l'île de Vancouver (Re) (ci-après " arrêt Colombie-Britannique c. Canada "10;

gence ou divergence? ", (2008) 49-2 C. de D. 297, a soutenu que le principe de l'interprétation large et libérale ainsi que la méthode d'interprétation contextuelle étaient deux composantes d'une seule et même approche, soit la méthode téléologique. Dans le présent texte, nous mettrons en opposition l'interprétation téléologique, ou finaliste, et l'interprétation littérale et technique.

4. In re The Initiative and Referendum Act, [1919] A.C. 935.

5. $\quad$ Procureur général du Québec c. Blaikie et autres, [1979] 2 R.C.S. 1016.

6. $\quad$ Procureur général du Manitoba c. Forest, [1979] 2 R.C.S. 1032.

7. Renvoi : Compétence du Parlement relativement à la Chambre haute, [1980] 1 R.C.S. 54.

8. Le procureur général de l'Ontario c. SEFPO, [1987] 2 R.C.S. 2.

9. New Brunswick Broadcasting Co c. Nouvelle-Écosse (Président de l'Assemblée législative), [1993] 1 R.C.S. 319.

10. Colombie-Britannique (Procureur général) c. Canada (Procureur général); Acte concernant le chemin de fer de l'̂̂le de Vancouver (Re), [1994] 2 R.C.S. 41. 
- Renvoi relatif à la rémunération des juges de la Cour provinciale de I.P.E.; Renvoi relatif à l'indépendance et à l'impartialité des juges de la Cour provinciale de I.P.E. (ciaprès "Renvoi relatif aux juges de la Cour provinciale ") ${ }^{11}$

- Hogan v. Newfoundland (Attorney General) (1998) (ci-après " arrêt Hogan 1998 "); Hogan v. Newfoundland (Attorney General) (1999) (ci-après " arrêt Hogan 1999"); Hogan v. Newfoundland (Attorney General) (2000) (ci-après " arrêt Hogan CA ") ${ }^{12}$;

- Renvoi relatif à la sécession du Québec ${ }^{13}$;

- Potter c. Quebec (Procureur général) (ci-après " arrêt Potter $C S$ ॥); Potter c. Québec (Procureur général) (ci-après " arrêt Potter CA "14;

- Renvoi relatif à la Loi sur la Cour suprême, art. 5 et 6 (ci-après "Renvoi relatif à la Loi sur la Cour suprême ")15;

- Renvoi relatif à la réforme du Sénat ${ }^{16}$.

\section{A) Le Renvoi relatif à The Initiative and Referendum Act}

Dans le Renvoi relatif à The Initiative and Referendum Act, le Comité judiciaire du Conseil privé a jugé que la province du Manitoba ne pouvait pas édicter que les lois provinciales pourraient être faites et révoquées par suite d'un vote direct des électeurs plutôt qu'uniquement par le truchement de l'assemblée législative provinciale $^{17}$. Plus particulièrement, le vicomte Haldane a affirmé,

11. Renvoi relatif à la rémunération des juges de la Cour provinciale de I.P.E.; Renvoi relatif à l'indépendance et à l'impartialité des juges de la Cour provinciale de I.P.E., [1997] 3 R.C.S. 3.

12. Hogan v. Newfoundland (Attorney General) (1998), 156 D.L.R. (4th) 139; Hogan v. Newfoundland (Attorney General) (1999), 173 Nfld. \& PEIR 148; Hogan v. Newfoundland (Attorney General), (2000) 189 Nfld. \& PEIR 183.

13. Renvoi relatif à la sécession du Québec, [1998] 2 R.C.S. 217.

14. Potter c. Quebec (Procureur général), 1998 CanLII 9495 (QC CS); Potter c. Québec (Procureur général), [2001] R.J.Q. 2823.

15. Renvoi relatif à la Loi sur la Cour suprême, art. 5 et 6, 2014 CSC 21.

16. Renvoi relatif à la réforme du Sénat, 2014 CSC 32.

17. La loi intitulée "The Initiative and Referendum Act " est décrite dans la décision du Comité judiciaire du Conseil privé : Renvoi relatif à The 
au nom du tribunal, qu'une province ne pouvait pas modifier la position du lieutenant-gouverneur en vertu de l'article 92 (1) de la Loi constitutionnelle de 1867 (ci-après "Loi de 1867 ")18. Or, en l'espèce, selon le vicomte Haldane, la loi manitobaine contestée portait effectivement atteinte à des droits importants relativement à la théorie légale propre à la fonction de lieutenant-gouverneur ${ }^{19}$.

Le vicomte Haldane a également affirmé, quoique de façon incidente, que la législature provinciale ne pouvait pas créer un nouvel organe législatif qui n'est pas mentionné dans la Loi de 186720. Dans l'arrêt $S E F P O^{21}$, le juge Beetz a dit de cette opinion

Initiative and Referendum Act, préc., note 4, 939-941. À la page 944 de sa décision, cette instance déclare ladite loi inconstitutionnelle.

18. Id., 943: "The references their Lordships have already made to the character of the office of Lieutenant-Governor, and to his position as directly representing the Sovereign in the province, renders natural the exclusion of his office from the power conferred on the Provincial Legislature to amend the constitution of the Province. " Notons au passage que l'article 92 (1) de la Loi constitutionnelle de 1867 (R.-U.), 30 \& 31 Vict., c 3, reproduite dans L.R.C. 1985, app. II, no 5 conférait aux provinces le pouvoir de modifier unilatéralement, par simple loi, leur constitution respective, sauf les dispositions relatives à la charge de lieutenantgouverneur. Cet article a été abrogé en 1982 et remplacé par l'article 45 de la Loi de 1982, lequel prévoit désormais ce qui suit : "Sous réserve de l'article 41, une législature a compétence exclusive pour modifier la constitution de sa province. " Nous reviendrons plus bas sur le remplacement de l'article 92 (1) de la Loi de 1867 par l'article 45 de la Loi de 1982.

19. Renvoi relatif à The Initiative and Referendum Act, id., 944 :

Their Lordships are of opinion that the language of the Act cannot be construed otherwise than as intended seriously to affect the position of the Lieutenant-Governor as an integral part of the Legislature, and to detract from rights which are important in the legal theory of that position [...] Thus the Lieutenant-Governor appears to be wholly excluded from the new legislative authority. Parmi les pouvoirs qui font partie intégrante de la fonction de lieutenantgouverneur, notons ceux qui consistent à sanctionner les projets de loi, à convoquer et à dissoudre les assemblées législatives de même qu'à réserver les projets de loi pour la signification du bon plaisir du gouverneur général.

20. Id., 945 : " but it does not follow that it [the Legislature of a Province] can create and endow with its own capacity a new legislative power not created by the Act to which it owes its own existence ".

21. Nous reviendrons sur cette décision plus bas. 
incidente qu'elle pouvait étayer la proposition plus générale selon laquelle le pouvoir de modification constitutionnelle que l'article 92 (1) de la Loi de 1867 accordait aux provinces ne comprenait pas nécessairement celui de provoquer des bouleversements constitutionnels profonds par l'introduction d'institutions politiques étrangères et incompatibles avec le système canadien ${ }^{22}$.

Dès lors, la question se pose de savoir ce que signifie au juste l'interprétation que le juge Beetz a faite dans l'arrêt SEFPO de l'opinion incidente du vicomte Haldane. Cela est dur à dire. Minimalement, cette interprétation signifie que les provinces ne peuvent pas se servir de leurs pouvoirs unilatéraux en matière de modification constitutionnelle pour remplacer leur assemblée législative par une autre instance ni pour instaurer le vote direct des électeurs.

Il ressort néanmoins de tout cela que le Renvoi relatif à The Initiative and Referendum Act comporte sa part d'ambiguïté en ce qui concerne la portée des pouvoirs unilatéraux provinciaux en matière de modification constitutionnelle. Et cela, c'est sans compter qu'il ne dit rien en ce qui a trait aux pouvoirs unilatéraux fédéraux en pareille matière.

22. Sur ce point, voir l'arrêt SEFPO, préc., note 8, par. 111. Le fait que le Québec ne peut pas - à l'instar des autres provinces - provoquer des bouleversements constitutionnels profonds dans son système politique ne l'a toutefois pas empêché d'abolir son conseil législatif en 1968. En effet, dans l'affaire Montplaisir c. Québec (Procureur général), [1997] R.J.Q. 109, le juge Pierre Béliveau, de la Cour supérieure du Québec, a conclu, à bon droit, qu'une législature provinciale (celle du Québec en l'occurrence) pouvait unilatéralement abolir son conseil législatif, puisque l'existence d'un système à deux chambres au niveau provincial n'était pas l'un des principes fondamentaux que la Constitution de 1867 voulait protéger à tout prix. Selon le juge Béliveau, l'abolition du Conseil législatif du Québec, en vertu de la Loi concernant le Conseil législatif, S.Q. 1968, c. 9, ne portait pas atteinte à la charge de lieutenant-gouverneur et avait été faite de façon régulière. 
Parlant justement des pouvoirs fédéraux, certains pourraient être tentés de soutenir que le Parlement ${ }^{23}$ ne peut pas, à l'instar des provinces, provoquer des bouleversements constitutionnels profonds qui soient incompatibles avec le système canadien. Sans vouloir nous étendre trop longuement sur ce sujet qui mériterait de toute façon une analyse détaillée à lui seul, permettons-nous d'affirmer qu'il est effectivement fort possible que ce qui a été dit dans le Renvoi relatif à The Initiative and Referendum Act au sujet des pouvoirs provinciaux unilatéraux de modification constitutionnelle s'applique aussi au Parlement.

De toute façon, les pouvoirs unilatéraux fédéraux de modification constitutionnelle sont déjà fort limités ${ }^{24}$, puisqu'ils ne

23. Dans le présent texte, le mot "Parlement", employé seul, désigne le Parlement du Canada.

24. Avant le rapatriement de la Constitution canadienne, les pouvoirs unilatéraux de modification constitutionnelle dont jouissait le Parlement découlaient de l'article 91 (1) de la Loi de 1867. En effet, cette disposition - adoptée en 1949 - conférait au Parlement le pouvoir suivant :

[Édicter des lois relativement à la] modification, de temps à autre, de la constitution du Canada, sauf en ce qui concerne les matières rentrant dans les catégories de sujets que la présente loi attribue exclusivement aux législatures des provinces, ou en ce qui concerne les droits ou privilèges accordés ou garantis, par la présente loi ou par toute autre loi constitutionnelle, à la législature ou au gouvernement d'une province, ou à quelque catégorie de personnes en matière d'écoles, ou en ce qui regarde l'emploi de l'anglais ou du français, ou les prescriptions portant que le parlement du Canada tiendra au moins une session chaque année et que la durée de chaque chambre des communes sera limitée à cinq années, depuis le jour du rapport des brefs ordonnant l'élection de cette chambre; toutefois, le parlement du Canada peut prolonger la durée d'une chambre des communes en temps de guerre, d'invasion ou d'insurrection, réelles ou appréhendées, si cette prolongation n'est pas l'objet d'une opposition exprimée par les votes de plus du tiers des membres de ladite chambre.

L'article 91 (1) en cause a été abrogé en 1982 et remplacé par l'article 44 de la Loi de 1982, lequel prévoit ce qui suit : "Sous réserve des articles 41 et 42 , le Parlement a compétence exclusive pour modifier les dispositions de la Constitution du Canada relatives au pouvoir exécutif fédéral, au Sénat ou à la Chambre des communes. " Nous reviendrons plus bas sur le 
peuvent pas être exercés à l'égard de sujets qui sont soumis à une modalité complexe de modification constitutionnelle, comme la charge de reine et de gouverneur général ${ }^{25}$, le principe de la représentation proportionnelle des provinces à la Chambre des communes ${ }^{26}$, le droit d'une province d'avoir à la Chambre des communes un nombre de députés au moins égal à celui des sénateurs par qui elle était habilitée à être représentée lors de l'entrée en vigueur de la partie V de la Loi de $1982^{27}$, les pouvoirs du Sénat ${ }^{28}$, le mode de sélection des sénateurs ${ }^{29}$, le nombre de sénateurs par province ${ }^{30}$, les conditions de résidence que les sénateurs doivent remplir ${ }^{31}$, les autres caractéristiques essentielles du Sénat ${ }^{32}$, de même que le parlementarisme et le principe du gouvernement responsable ${ }^{33}$.

remplacement de l'article 91(1) de la Loi de 1867 par l'article 44 de la Loi de 1982.

25. Loi de 1982, art. 41 a).

26. Id., art. 42 (1) a). Dans l'affaire Campbell v. Canada (Attorney General), (1988) 49 D.L.R. (4th) 321, la Cour d'appel de la Colombie-Britannique a jugé que le Parlement pouvait unilatéralement modifier l'article 51 de la Loi de 1867 en vertu de l'article 44 de la Loi de 1982, mais qu'il ne pouvait pas, ce faisant, toucher le principe de la représentation proportionnelle des provinces à la Chambre des communes. En effet, la modification de ce principe relève plutôt de la procédure 7/50 en vertu de l'article 42 (1) a) de la Loi de 1982.

27. Loi de 1982, art. 41 b).

28. Id., art. $42(1)$ b).

29. Id., art. $41(1)$ b).

30. Id., art. 42 (1) c).

31. Id.

32. Voir sur ce point le Renvoi relatif à la Chambre haute, préc., note 7 , et le Renvoi relatif à la réforme du Sénat, préc., note 16, où la Cour suprême a jugé que les caractéristiques essentielles du Sénat devaient échapper aux pouvoirs unilatéraux fédéraux de modification constitutionnelle.

33. Sur la possibilité que le parlementarisme de type britannique et le principe du gouvernement responsable soient "enchâssés " dans la Constitution du Canada, voir l'arrêt SEFPO, préc. note 8 , par. 85 et 111 , où le juge Beetz, au nom de ses collègues McIntyre, Le Dain et La Forest, a déclaré ce qui suit :

La plus fondamentale d'entre elles est probablement le principe du gouvernement responsable qui est en grande partie non écrit, bien qu'il soit mentionné implicitement dans le préambule de la Loi constitutionnelle de 1867. [...] 


\section{B) L'arrêt Blaikie}

Dans l'arrêt Blaikie, la Cour suprême du Canada a eu à se prononcer sur la capacité de la législature ${ }^{34}$ québécoise de modifier unilatéralement les dispositions de l'article 133 de la Loi de 1867 (cette dernière étant appelée Acte de l'Amérique du Nord britannique au moment où l'arrêt a été rendu). Plus particulièrement, cet arrêt portait sur la constitutionnalité des articles 7 à 13 de la Charte de la langue française ${ }^{35}$, traitant de la langue de la justice et de la législation au Québec.

La Loi de 1867] ne comprend pas nécessairement le pouvoir de provoquer des bouleversements constitutionnels profonds par l'introduction d'institutions politiques étrangères et incompatibles avec le système canadien.

Cela a fait dire aux auteurs Henri BRUn, Guy TREMBLAY et Eugénie Broulllet, Droit constitutionnel, 6e éd., Cowansville, Éditions Yvon Blais, 2014, no IV.121, que cette approche " empêcherait le Québec, par exemple, de remplacer son régime parlementaire, son système de gouvernement responsable, par une séparation des pouvoirs à l'américaine ". Voir également l'affaire Figueroa c. Canada (Procureur général), 2003 CSC 37, par. 155, où le juge LeBel a écrit ceci :

La notion de gouvernement majoritaire reste ainsi étroitement liée à la tradition canadienne de gouvernement responsable, puisqu'un parti donné, dirigé par un chef unique dont on connaît l'identité, doit répondre des politiques gouvernementales (MacIvor, loc. cit., p. 29). Je ne prétends pas par là, je tiens à le préciser, qu'un gouvernement responsable ne saurait exister lorsqu'un gouvernement de coalition ou minoritaire est élu. J'affirme seulement qu'on peut raisonnablement considérer que, dans notre système politique particulier, les gouvernements majoritaires présentent certains avantages à cet égard.

Quant au mot "enchâssement " et au verbe "enchâsser ", ils font partie du jargon constitutionnel canadien. Ils désignent le fait d'inscrire, d'incorporer ou d'intégrer dans la Constitution.

34. Dans le présent texte, nous emploierons le terme "législature " pour désigner les assemblées législatives des provinces.

35. Charte de la langue française, L.R.Q. 1977, c. C-11. Les articles 7 à 13 constituaient le chapitre III du titre premier de cette charte. Ils étaient manifestement incompatibles avec les dispositions de l'article 133 de la Loi de 1867, ce que la Cour suprême a d'ailleurs facilement reconnu à la page 1022 de l'arrêt Blaikie, préc., note 5. 
L'élément en jeu essentiellement était l'interprétation de l'article 92 (1) de la Loi de 1867. La Cour suprême a alors affirmé, à l'unanimité, que la modification de la partie québécoise de l'article 133 ne saurait d'aucune façon relever de l'article 92 (1) en question. Pour tirer cette conclusion, la Cour suprême s'est fondée sur " des motifs qui transcendent l'interprétation la plus large du par. 92 (1), motifs qui ont été exposés de façon convaincante dans le jugement du juge en chef Deschênes et adoptés par la Cour d'appel du Québec 36 ". Ces motifs puisaient leur source dans l'histoire et dans le fait que l'article 133 faisait "partie indivisiblement de la constitution du Canada et du Québec en donnant au français et à l'anglais un statut officiel au Parlement, devant les tribunaux du Canada, de même qu'à la législature et devant les tribunaux du Québec 37 ". Tout compte fait, sur les questions de détails et d'histoire, la Cour suprême a tout simplement fait siens " les motifs du juge en chef Deschênes renforcés par ceux de la Cour d'appel du Québec 38 ".

La Cour suprême a poursuivi en disant ceci de l'article 133 :

[C'est] une disposition intangible qui non seulement interdit au Parlement et à la législature du Québec de la modifier unilatéralement mais assure également aux membres du Parlement ou de la législature du Québec et aux plaideurs devant les tribunaux du Canada ou du Québec le droit d'utiliser le français ou l'anglais dans les délibérations du Parlement ou de l'Assemblée législative et dans les procédures (y compris les plaidoiries orales) devant les tribunaux du Canada ou du Québec ${ }^{39}$.

Par conséquent, la Cour suprême en est venue à la conclusion que les articles de la Charte de la langue française qui étaient contestés se trouvaient ultra vires de la législature québécoise.

36. Arrêt Blaikie, préc., note 5, 1025.

37. Id.

38. Id., 1027.

39. Id., 1026-1027. 
Dans l'arrêt Blaikie, la Cour suprême (et avant elle, la Cour supérieure et la Cour d'appel du Québec ${ }^{40}$ ) s'est donc fondée à la fois sur l'esprit de l'article 133 de la Loi de 1867 et sur sa signification historique, pour faire échapper ce dernier à la portée de l'article 92 (1) de cette loi. Or, il est bien établi que celui-ci a été remplacé par l'article 45 de la Loi de 1982, qui joue maintenant le même rôle que le faisait l'article 92 (1) de la Loi de 186741. C'est donc dire que la jurisprudence qui était applicable audit article 92 (1) - dont l'arrêt Blaikie - l'est aussi à l'article 45 de la Loi de 1982.

Par conséquent, il est parfaitement clair que l'article 45 de la Loi de 1982 ne doit porter que sur des modifications qui, tant dans

40. Dans l'arrêt Blaikie, la Cour supérieure et la Cour d'appel du Québec ont toutes deux conclu que l'histoire de l'article 133 de la Loi de 1867 indiquait que celui-ci n'était pas divisible entre sa partie provinciale et sa partie fédérale. Il s'agissait plutôt d'une disposition intangible, qui participait de la Constitution du Canada et qui, de ce fait, était hors de portée de toute intervention législative unilatérale du Parlement ou de la législature québécoise. Sur ce point, voir les affaires Blaikie c. PG du Québec, [1978] C.S. 37, et Blaikie c. PG du Québec, [1978] C.A. 351.

41. Renvoi relatif à la réforme du Sénat, préc., note 16, par. 46. La Cour suprême y a affirmé que les articles 44 et 45 de la Loi de 1982 "jouent essentiellement le même rôle que jouaient les par. 91(1) et 92(1) de la Loi de 1867 qui ont été abrogés lors de l'adoption de la Loi constitutionnelle de 1982: Accord constitutionnel: Projet canadien de rapatriement de la Constitution, notes explicatives 7 et 8 ". Puis, au paragraphe 48, la Cour suprême a dit ce qui suit :

Les articles 44 et 45, qui ont remplacé ces dispositions, accordent au Parlement fédéral et aux législatures provinciales la capacité de modifier unilatéralement certains éléments de la Constitution qui concernent leur propre ordre de gouvernement mais qui ne mettent pas en cause les intérêts des autres ordres de gouvernement. Ce pouvoir limité d'effectuer des changements unilatéralement reflète le principe selon lequel le Parlement et les provinces sont des acteurs égaux dans la structure constitutionnelle canadienne. Aucun des ordres de gouvernement, agissant seul, ne peut modifier les nature et rôle fondamentaux des institutions créées par la Constitution. Toutefois, ces institutions peuvent être maintenues et même changées jusqu'à un certain point en vertu des art. 44 et 45 , à condition que leur nature et leur rôle fondamentaux demeurent intacts. 
leur esprit que dans leur libellé, ne concernent que la constitution interne de la province, en ce qu'elles ne visent que l'organisation et le fonctionnement des institutions provinciales, ne mettent d'aucune façon en cause les intérêts des autres partenaires fédératifs et ne touchent pas aux conditions fondamentales de l'union fédérale. Nous verrons ci-dessous, lorsque nous examinerons le Renvoi relatif à la Chambre haute, que l'article $44 \mathrm{de}$ la Loi de 1982 - qui, rappelons-le, a pris la place de l'ancien article 91 (1) de la Loi de 1867 lorsqu'est survenu le rapatriement de la Constitution canadienne - doit être interprété et appliqué de la même façon que l'article 45 susmentionné.

\section{C) L'arrêt Forest}

Dans l'arrêt Forest, on était en présence d'une contestation d'une loi manitobaine datant de 1890, An Act to Provide that the English Language shall be the Official Language of the Province of Manitoba ${ }^{42}$, pour cause d'incompatibilité avec l'article 23 de la loi suivante : An Act to amend and continue the Act 32 and 33 Victoria, chapter 3; and to establish and provide for the Government of the Province of Manitoba (ci-après "Loi de 1870 sur le Manitoba")43. La Cour suprême a effectivement déclaré la loi manitobaine de 1890 invalide et s'est alors exprimée comme suit :

Il n'est pas nécessaire de rechercher en l'espèce si cette disposition législative emporte restriction du pouvoir de modification qui découle du par. 92 (1) par application de l'art. 2 de l'Acte du Manitoba. Il suffit de noter que, quelle que soit l'interprétation qu'on lui donne, elle ne peut certainement pas avoir pour effet de donner à la législature du Manitoba à l'égard de l'art. 23 de l'Acte du Manitoba un pouvoir de modification que le Québec n'a pas à l'égard de l'art. $133^{44}$.

42. An Act to Provide that the English Language shall be the Official Language of the Province of Manitoba, R.S.M. 1970, c. O10.

43. An Act to amend and continue the Act 32 and 33 Victoria, chapter 3; and to establish and provide for the Government of the Province of Manitoba, S.C. 1870 , c. 3, reproduit dans LRC 1985, ann. II, $\mathrm{n}^{\circ} 8$.

44. Arrêt Forest, préc., note 6, 1039. 
Il faut dire que l'article 23 en question reprenait - en les rendant précisément applicables au Manitoba - les termes de l'article 133 de la Loi de 186745. Selon la Cour suprême, il n'y avait donc pas lieu d'interpréter l'article 23 différemment de l'article 133. Ainsi, les deux articles se devaient d'échapper à la portée de l'article 92 (1) de la Loi de 1867.

Finalement, on retiendra des arrêts Blaikie et Forest, pour ce qui est de la question précise de la modification constitutionnelle, que l'application ou non de l'article 92 (1) de la Loi de 1867 dépendait à l'époque non seulement du libellé de la modification envisagée, mais aussi de l'esprit de cette dernière. Dans ces deux arrêts, la Cour suprême a donné à cet article une interprétation que l'on pourrait qualifier de téléologique plutôt que de purement technique. Or, de nos jours, comme nous l'avons vu ci-dessus, c'est exactement la même interprétation qu'il convient de donner à l'article 45 de la Loi de 1982.

\section{D) Le Renvoi relatif à la Chambre haute}

En 1978, le gouvernement canadien a déposé au Parlement le projet de loi sur la Loi modifiant la Constitution du Canada dans certains domaines ressortissant à la compétence législative du Parlement du Canada et prévoyant les mesures nécessaires à la modification de la Constitution dans certains autres domaines (ciaprès "projet de loi no C-60")46, lequel devait, entre autres,

45. La Cour suprême, dans l'arrêt Forest, préc., note 6, à la p. 1036, a noté "l'étroite ressemblance " entre l'article 23 de la Loi de 1870 sur le Manitoba et l'article 133 de la Loi de 1867.

46. Loi modifiant la Constitution du Canada dans certains domaines ressortissant à la compétence législative du Parlement du Canada et prévoyant les mesures nécessaires à la modification de la Constitution dans certains autres domaines, projet de loi $\mathrm{n}^{\circ} \mathrm{C}-60$ (1978), 3e sess., 30e légis. (Can.), art. 62-70. Voici ce que le professeur André TREmBlay, La réforme de la Constitution au Canada, Montréal, Éditions Thémis, 1995, p. 60, a affirmé au sujet de ce projet de loi :

Le gouvernement fédéral déposa aux Communes le 12 juin 1978 un document intitulé Le temps d'agir, lequel fut suivi quelques jours plus tard $\mathrm{du}$ dépôt du Projet de loi sur la réforme 
permettre de réformer le Sénat en modifiant la répartition des sièges entre les régions, en remplaçant le veto absolu dont celui-ci disposait dans le processus législatif fédéral par un simple veto suspensif et en confiant aux provinces le pouvoir de choisir un certain nombre de sénateurs ${ }^{47}$.

Devant les réactions négatives que le projet de loi no $\mathrm{C}-60$ a suscitées, le gouvernement fédéral a demandé à la Cour suprême, dans le contexte d'un renvoi, si le Parlement pouvait procéder unilatéralement à une telle réforme de la Chambre haute, en vertu de l'article 91 (1) de la Loi de 186748.

constitutionnelle (C-60) qui contenait un ensemble fort complexe de propositions de renouvellement ou de canadianisation de la Constitution. Avec le premier document, le gouvernement concrétisait l'annonce faite en octobre 1977, lors du discours du trône, de reprendre l'initiative des discussions constitutionnelles. Il ne posait que deux préalables au renouvellement de la fédération : le premier étant le maintien de la fédération et le second, l'inclusion d'une Charte des droits dans la Constitution. $[\ldots]$

Les propositions détaillées que le gouvernement annonçait pour "bientôt" devaient "donner aux parlementaires, aux gouvernements provinciaux ainsi qu'aux citoyens l'occasion de les étudier et d'en débattre ". On trouve ces propositions détaillées dans le projet de loi $\mathrm{C}-60$, Projet de loi sur la réforme constitutionnelle, dont la première lecture eut lieu aux Communes le 20 juin 1978. Le projet de loi représente un excellent modèle d'initiative constitutionnelle voué au départ à l'échec.

47. Notons que, en vertu du projet de loi no C-60, le Sénat aurait été transformé en une "chambre de la fédération ".

48. Dans le Renvoi relatif à la Chambre haute, préc., note 7 , les questions soumises à la Cour suprême par le gouverneur général en conseil portaient sur l'abolition du Sénat, sur le changement de nom de cette institution, sur la modification du nombre et du pourcentage de sénateurs pour chaque province ou territoire, sur la modification des qualités requises pour être membre du Sénat, sur la modification de la durée du mandat des sénateurs, sur la modification du processus de nomination des sénateurs et sur l'octroi d'un simple veto suspensif au Sénat concernant l'adoption des lois fédérales. La Cour suprême n'a pas été en mesure de répondre à toutes les questions posées, faute de précisions sur le contexte qui lui aurait permis de formuler une réponse catégorique ou satisfaisante. 
La Cour suprême a alors conclu, à l'occasion du Renvoi relatif à la Chambre haute, que l'article 91 (1) en question autorisait certes le Parlement à modifier " la constitution du gouvernement fédéral dans les matières qui concernent uniquement ce gouvernement 49 ", mais qu'il ne lui conférait toutefois pas le pouvoir d'apporter unilatéralement des changements constitutionnels tels que l'abolition du Sénat ou la modification de ses caractéristiques essentielles ou fondamentales, étant donné que ce type de modification mettait en cause les intérêts des provinces tout autant que ceux de l'ordre fédéral de gouvernement ${ }^{50}$.

Dans le Renvoi relatif à la Chambre haute, la Cour suprême en est donc venue à la conclusion que l'application ou non de l'article 91 (1) de la Loi de 1867 devait dépendre de la portée technique de la modification envisagée et aussi de la nature de cette dernière. Ainsi, lorsque la modification en question concernait, tant dans son esprit que dans son libellé, les relations fédéralesprovinciales ou le compromis fédératif, elle ne pouvait alors pas être accomplie unilatéralement par le Parlement en vertu de l'article 91 (1); il fallait plutôt, pour la concrétiser, faire appel au Parlement britannique ${ }^{51}$.

Dans le Renvoi relatif à la Chambre haute, la Cour suprême a donc donné à l'article 91 (1) de la Loi de 1867 la même

49. Id., 71. Notons au passage que, parmi les caractéristiques essentielles ou fondamentales du Sénat qui se devaient d'échapper à la portée de l'article 91 (1) de la Loi de 1867, la Cour suprême du Canada a indiqué les suivantes : l'abolition du Sénat, la transformation du droit de veto absolu que possède le Sénat concernant l'adoption des lois fédérales en un simple veto suspensif, l'élection directe des sénateurs par la population ainsi que la modification du nombre de sénateurs par province et de leur pourcentage.

50. Id., 74 et suiv. Soulignons que, à la suite du Renvoi relatif à la Chambre haute, préc., note 7 , le gouvernement canadien a abandonné le projet de loi no $\mathrm{C}-60$.

51. Le recours au Parlement britannique pour modifier la Constitution du Canada, dans ses parties les plus substantielles, se fondait alors sur l'article 7 (1) et l'article 4 du Statut de Westminster de 1931, 22 Geo V, c. 4 (R.-U.), reproduit dans LRC 1985, ann. II, no 27. 
interprétation téléologique qu'elle avait attribuée à l'article 92 (1) dans les arrêts Blaikie et Forest. Depuis le remplacement de l'article 91 (1) par l'article 44 de la Loi de 1982, remplacement survenu à l'occasion du rapatriement de la Constitution canadienne, comme nous l'avons vu ci-dessus, il y a lieu d'interpréter ce dernier article de la même façon que l'ancien article 91 (1). Dès lors, l'interprétation à conférer désormais à l'article 44 de la Loi de 1982 coïncide avec celle qu'il convient aussi de donner à son article $45^{52}$.

Notons au passage que l'une des différences fondamentales entre le Renvoi relatif à la Chambre haute et les arrêts Blaikie et Forest tenait évidemment au fait que le premier portait sur l'article 91 (1) de la Loi de 1867, alors que les deux arrêts concernaient son article 92 (1). Cependant, au-delà de cette différence, il y a énormément de parenté entre toutes ces décisions. En effet, dans les trois cas, la Cour suprême a interprété la portée du pouvoir de modification constitutionnelle en cause (soit les articles 91 (1) et 92 (1) de la Loi de 1867) en tenant compte non seulement du libellé, mais aussi - et peut-être même surtout - de la nature de la ou des modifications examinées.

Ainsi, dans l'arrêt Blaikie, la disposition modifiée (bien qu'elle l'ait été tacitement et indirectement) était la partie québécoise de l'article 133 de la Loi de 1867. La Cour suprême a alors déclaré qu'une telle modification ne pouvait pas être effectuée par la législature québécoise en vertu de l'article 92 (1), étant donné le rôle clé qu'avait joué l'ensemble de l'article 133 dans le compromis fédératif de 1867 et l'impossibilité (selon la Cour suprême) de séparer unilatéralement la partie québécoise de cet article de sa partie fédérale. Dans l'arrêt Forest, c'était l'article 23 de la Loi de 1870 sur le Manitoba que la législature manitobaine voulait modifier (tacitement et indirectement là encore). La Cour suprême a fait

52. Cela est dit sous réserve évidemment du fait que l'article 44 de la Loi de 1982 accorde un pouvoir de modification constitutionnelle à la Chambre des communes et au Sénat (ce dernier n'ayant cependant qu'un veto suspensif de 180 jours, en vertu de l'article 47 de la Loi de 1982), alors que l'article 45 de cette loi octroie le même type de pouvoir à chaque législature. 
échapper cette modification à la portée de l'article 92 (1) après avoir établi un parallèle entre l'article 23 de la Loi de 1870 sur le Manitoba et l'article 133 de la Loi de 1867.

Enfin, dans le Renvoi relatif à la Chambre haute, les modifications envisagées par le gouvernement canadien portaient sur le Sénat. La Cour suprême a d'abord établi une distinction entre les caractéristiques essentielles et les caractéristiques non essentielles (ou secondaires) de cette institution, puis elle a affirmé que les premières touchaient aux intérêts provinciaux, contrairement aux secondes. Par conséquent, la Cour suprême a conclu que seules les caractéristiques non essentielles du Sénat pouvaient être modifiées en vertu de l'article 91 (1) de la Loi de 1867. De leur côté, les caractéristiques essentielles du Sénat ne pouvaient, à l'époque où le Renvoi relatif à la Chambre haute a été rendu, être modifiées que par le Parlement de Westminster. Aujourd'hui, ces caractéristiques relèvent de modalités de modification constitutionnelle dites complexes, comme nous le verrons en détail plus bas.

\section{E) L'arrêt SEFPO}

Dans l'arrêt SEFPO, la Cour suprême devait se prononcer sur la validité des articles 12 à 16 d'une loi ontarienne, soit The Public Service $A c t^{53}$, qui avait pour objet d'interdire aux fonctionnaires provinciaux et aux employés de Sa Majesté aux droits de l'Ontario d'exercer certaines activités politiques au niveau fédéral.

Le juge Beetz, au nom de ses collègues les juges McIntyre, Le Dain et La Forest54, s'est étendu longuement sur le sens qu'il

53. The Public Service Act, R.S.O. 1980, c. 418.

54. Six juges seulement ont pris part à cet arrêt. Le juge Lamer s'est dit d'accord avec le juge Beetz. 
convenait de donner aux mots "constitution de la province " dans l'article 92 (1) de la Loi de 1867. Il a alors affirmé ce qui suit :

[L]es dispositions relatives à la constitution de l'état fédéral prises dans leur ensemble, ou essentielles à la mise en œuvre du principe fédéral échappent au pouvoir de modification que le par. 92 (1) accorde à la province.

$[\ldots]$

En outre, d'autres dispositions de la Loi constitutionnelle de 1867 ont pu même être intangibles et considérées comme échappant au par. 92 (1), non pas parce qu'elles étaient essentielles à la mise en œuvre du principe fédéral, mais parce que, pour des raisons historiques, elles constituaient une condition fondamentale de l'union formée en 186755 .

Puis, après avoir renvoyé à l'arrêt Blaikie rendu par la Cour suprême, le juge Beetz s'est exprimé comme suit :

[U]ne disposition peut généralement être considérée comme une modification de la constitution d'une province lorsqu'elle porte sur le fonctionnement d'un organe du gouvernement de la province, pourvu qu'elle ne soit pas par ailleurs intangible parce qu'indivisiblement liée à la mise en œuvre du principe fédéral ou à une condition fondamentale de l'union 56 .

Enfin, le juge Beetz a conclu que les dispositions de la loi ontarienne qui étaient contestées ne violaient pas une "condition fondamentale de l'union comme c'était le cas dans l'affaire Blaikie 57 ", qu'elles n'étaient pas non plus "incompatibles avec la mise en œuvre du principe fédéral ${ }^{58}$ ", qu'elles constituaient " une modification législative ordinaire de la constitution de l'Ontario au sens du par. 92 (1) de la Loi constitutionnelle de 186759 " et qu'elles

55. Arrêt SEFPO, préc., note 8, par. 88-89.

56. Id., par. 90.

57. Id., par. 102 .

58. Id., par. 103.

59. Id., par. 107. 
ne touchaient "aux élections fédérales et provinciales que d'une manière accessoire 60 ".

Aux fins de notre propos, nous retiendrons de l'arrêt SEFPO que les dispositions constitutionnelles qui représentaient initialement une condition fondamentale de l'union canadienne devaient échapper à la portée de l'article 92 (1) de la Loi de 1867 et qu'il en était de même pour les dispositions liées à la mise en œuvre du principe fédéral.

C'est donc dire que, à l'instar de l'arrêt Blaikie, l'arrêt SEFPO a consacré l'idée voulant que, dans l'interprétation du pouvoir de modification découlant de l'article 92 (1) de la Loi de 1867, il fallait tenir compte de l'esprit de la disposition modifiée, de son sens historique, de son lien avec le principe fédératif et de son impact sur le système canadien.

L'arrêt SEFPO étaye également la proposition voulant que la Constitution canadienne possède une "structure constitutionnelle fondamentale 61 ", une "architecture interne " dira plus tard la Cour suprême dans le Renvoi relatif à la sécession du Québec ${ }^{62}$. Dans ce dernier avis, sur lequel nous reviendrons plus bas, la Cour suprême ajoutera que " [c]haque élément individuel de la Constitution est lié aux autres et doit être interprété en fonction de l'ensemble de sa structure 63 \%. Ces principes imprègnent la Constitution et lui donnent vie ${ }^{64}$.

\section{F) L'arrêt N.B. Broadcasting}

Dans l'arrêt N.B. Broadcasting, la Cour suprême a jugé, à la majorité, que les privilèges inhérents des organismes législatifs du Canada sont constitutionnalisés en vertu du préambule de la Loi de 1867.

60. Id., par. 151.

61. Id.

62. Renvoi relatif à la sécession du Québec, préc., note 13, par. 50 .

63. Id.

64. Id. 
La juge McLachlin a affirmé, au nom de trois de ses collègues, que les principes non écrits ont bel et bien leur place dans la Constitution canadienne. Bien qu'il existe au Canada un grand nombre de textes constitutionnels et bien qu'il ne convienne pas de transposer trop librement des concepts non écrits dans un régime constitutionnel qui a abouti à une constitution écrite, il n'y a pas lieu d'exclure de manière catégorique la reconnaissance de pouvoirs constitutionnels non écrits ou inexprimés ${ }^{65}$. Et la juge McLachlin de poursuivre ainsi :

Le professeur Hogg conclut que [TRADUCTION] " l'évolution progressive du Canada, du statut de colonie à celui d'un État, fait qu'il n'est pourvu d'aucun document constitutionnel complet " (p. 1-8). Il reconnaît que le texte du par. 52(2) ne constitue pas une preuve concluante que cette disposition constitue une définition exhaustive de la Constitution du Canada.

$[\ldots]$

Je suis d'accord avec l'essentiel de la pensée du professeur Hogg lorsqu'il fait observer que l'ajout d'autres textes aux 30 déjà énumérés à l'annexe mentionnée au par. 52(2) de la Loi constitutionnelle de 1982 risque d'avoir de graves conséquences compte tenu de la suprématie et de l'enchâssement prévus relativement à la "Constitution du Canada " aux par. 52(1) et 52(3). Toutefois, comme le reconnaît le professeur Hogg lui-même, le par. 52(2) ne se veut pas clairement exhaustif66.

Le juge La Forest a souscrit de façon générale aux motifs de la juge McLachlin. Le juge Cory, malgré sa dissidence, s'est dit, pour une grande part, d'accord avec cette dernière sur les motifs qu'elle avait rédigés.

Comme nous pouvons le constater, la Cour suprême a ouvert la porte à la possibilité que l'article 52 de la Loi de 1982 vise des

65. Sur ce point, voir l'arrêt N.B. Broadcasting, préc., note 9, 375-376.

66. Id., 377-378. 
textes autres que ceux qu'il énumère, voire des principes se dégageant de pareils textes ${ }^{67}$. L'article 52 ne se présente pas comme exhaustif, de rappeler la Cour suprême.

Dans le Renvoi relatif à la sécession du Québec, voici ce qu'a dit la Cour suprême au sujet de l'arrêt N.B. Broadcasting :

Notre Cour a indiqué dans New-Brunswick Broadcasting Co. c. Nouvelle-Écosse (Président de l'Assemblée législative), [1993] 1 R.C.S. 319, que les Canadiens reconnaissent depuis longtemps l'existence et l'importance des principes constitutionnels non écrits de notre système de gouvernement 68 .

L'arrêt N.B. Broadcasting appuie donc l'idée voulant que des privilèges constitutionnels inhérents, découlant du préambule de la Loi de 1867, fassent partie de la Constitution du Canada. Il s'agissait, en l'espèce, des pouvoirs constitutionnels non écrits et inexprimés qui étaient historiquement considérés comme nécessaires au bon fonctionnement des organismes législatifs. De là à affirmer que des principes constitutionnels sous-jacents sont susceptibles de faire partie de la définition de la Constitution du Canada au sens de l'article 52 (2) de la Loi de 1982, il n'y a qu'un pas que, comme nous le verrons plus bas, la Cour suprême a franchi dans sa jurisprudence ultérieure.

67. L'article 52 de la Loi de 1982, par l'emploi qui y est fait du mot " comprend ", ne se veut définitivement pas exhaustif. Il peut viser d'autres textes que ceux qui y sont explicitement mentionnés. Il peut aussi concerner des principes constitutionnels trouvant leur expression dans des textes autres que ceux qu'il énumère. Par exemple, dans le Renvoi relatif à la Loi sur la Cour suprême, préc., note 15, cette cour n'a pas voulu dire explicitement que la Loi sur la Cour suprême, L.R.C. 1985, c. S-26, était bel et bien enchâssée dans la Constitution du pays. Elle s'est contentée de préciser que les caractéristiques essentielles de la Cour l'étaient. Nous reviendrons sur ce point ci-dessous.

68. Renvoi relatif à la sécession du Québec, préc., note 13, par. 52. 


\section{G) L'arrêt Colombie-Britannique c. Canada}

Dans l'arrêt Colombie-Britannique c. Canada, le juge Iacobucci a tenu les propos suivants, au nom de la majorité :

Comme l'a reconnu le professeur Hogg dans Constitutional Law of Canada (3e éd. 1992), à la p. 9 :

[TRADUCTION] La définition de l'expression "Constitution du Canada " au par. 52(2) est précédée du terme " comprend ". En général, dans les lois canadiennes, le terme " comprend " indique que la définition n'est pas exhaustive. Le terme "désigne " précède habituellement une définition exhaustive. Cependant, compte tenu de la spécificité de la liste des textes législatifs et décrets, et des graves conséquences (savoir la primauté et la constitutionnalisation...) de l'inclusion d'autres instruments, il est certain qu'aucun tribunal n'aurait l'audace de faire des ajouts à la liste des 30 instruments énoncés dans l'annexe. En conséquence, il semble seulement réaliste de considérer la définition comme exhaustive, même si elle omet de nombreux instruments importants pour le gouvernement fédéral ou les gouvernements provinciaux. Par exemple, la définition ne mentionne pas les instruments antérieurs à $1867[\ldots]$.

L'argument de la Colombie-Britannique va à l'encontre de la notion de bon sens formulée par le professeur Hogg [...] Il n'est certes pas nécessaire d'écarter complètement la possibilité que des documents non énumérés au par. 52(2) de la Loi constitutionnelle de 1982 puissent quand même être considérés comme constitutionnels dans certains contextes. Cette question n'a pas à être tranchée en l'espèce ${ }^{69}$.

69. Arrêt Colombie-Britannique c. Canada, préc., note 10, 94. 
Comme on le voit, la Cour suprême n'a pas voulu se prononcer clairement sur la question de savoir si la "Constitution du Canada", au sens de l'article 52 (2) de la Loi de 1982, comprenait d'autres documents que ceux qui y sont expressément mentionnés. Elle n'a toutefois pas exclu une telle possibilité.

\section{H) Le Renvoi relatif aux juges de la Cour provinciale}

Dans le Renvoi relatif aux juges de la Cour provinciale, le juge en chef Lamer a affirmé, au nom de cinq de ses collègues, que l'indépendance de la magistrature découlait d'un principe structurel, incorporé par renvoi dans le droit canadien en vertu du préambule de la Loi de 1867. Sur l'article 52 (2) de la Loi de 1982, le juge Lamer a tenu les propos suivants :

Même si [...] je continue de douter que les privilèges des assemblées provinciales fassent partie de la Constitution [...], j'accepte le principe général que la Constitution comprend des règles non écrites - et écrites -, dans une large mesure sur le fondement du libellé du par. 52(2). En effet, comme notre Constitution est dérivée d'un régime constitutionnel dont les règles fondamentales ne sont pas fixées dans un seul document ou dans un ensemble de documents faisant autorité, il n'est pas étonnant qu'elle retienne certains aspects de cet héritage.

Toutefois, je tiens à ajouter une mise en garde. Comme je l'ai dit dans l'arrêt New Brunswick Broadcasting, précité, à la p. 355, 1'histoire constitutionnelle du Canada peut être considérée, en partie, comme une évolution " qui a abouti à la suprématie d'une constitution écrite définitive ". La préférence pour une Constitution écrite repose sur bon nombre de raisons importantes, particulièrement la certitude du droit et, par ce moyen, la légitimité du contrôle judiciaire fondé sur la Constitution. Compte tenu de ces préoccupations, qui sont au cœur de l'idée de constitutionnalisme, il est de la plus haute importance de préciser la source de ces normes non écrites. 
À mon avis, il est possible d'expliquer l'existence de bon nombre des règles non écrites de la Constitution canadienne en se reportant au préambule de la Loi constitutionnelle de $1867[\ldots]^{70}$.

Le juge La Forest, bien qu'il ait été dissident en partie, s'est dit d'accord avec le juge Lamer sur l'essentiel des motifs de ce dernier.

Donc, une fois de plus, la Cour suprême a reconnu que la Constitution canadienne comprenait des normes non écrites et que la définition qui se trouvait dans l'article 52 (2) de la Loi de 1982 n'était vraisemblablement pas exhaustive. La Cour suprême a ajouté toutefois que la reconnaissance de ces principes constitutionnels (ou structurels) ne devait pas devenir une invitation à négliger le texte écrit de la Constitution ${ }^{71}$.

\section{I) L'arrêt Hogan}

Dans une décision rendue le 8 janvier 1998, le juge Orsborn de la Cour supérieure de Terre-Neuve (Newfoundland Supreme Court, Trial Division) s'est prononcé, en tant que juge des requêtes interlocutoires, sur la validité d'une modification (alors envisagée) aux Conditions de l'union de Terre-Neuve au Canada, en vue de déconfessionnaliser le système scolaire de la province ${ }^{72}$. Plus précisément, des catholiques avaient allégué que cela constituait une modification de la Charte canadienne des droits et libertés (ci-

70. Renvoi relatif aux juges de la Cour provinciale, préc., note 11, par. 92-94.

71. Id., par. 93 et 104. Voir également le Renvoi relatif à la sécession du Québec, préc., note 13, par. 53.

72. Sur ce point, voir l'arrêt Hogan 1998, préc., note 12, par. 3. Les demandeurs cherchaient l'émission d'une injonction contre la proclamation par le gouverneur général du Canada de ce qui allait devenir la Modification constitutionnelle de 1998 (Terre-Neuve), TR/1998-25 (Gaz. Can. II), édition spéciale. 
après "Charte canadienne "73. Cet argument n'a pas été retenu par le juge Orsborn qui s'est, entre autres, exprimé comme suit :

Il est évident que, si un droit constitutionnel est adéquatement étendu ou restreint, la portée du contrôle judiciaire découlant de la Charte sera modifiée en conséquence. Toutefois, le fait que l'application de la Charte puisse être renforcée en raison d'une modification constitutionnelle qui retire à une province le pouvoir de discriminer ne signifie pas que la Charte elle-même ait été modifiée.

En l'espèce, la seule modification proposée est celle de l'article 17. Il n'y a pas un mot sur la modification de la Charte; le fait que l'application de cette dernière ait été élargie à Terre-Neuve n'équivaut pas à une modification visée par la partie V de la Loi constitutionnelle de $1982^{74}$.

73. Voir particulièrement l'arrêt Hogan 1998, préc., note 12, par. 100-102. Les demandeurs avaient également soutenu que l'article 93 de la Loi de 1867 s'appliquait à toutes les provinces - en raison de son paragraphe liminaire qui complète les articles 91 et 92 de la Loi de 1867 et qui confère aux provinces une compétence exclusive en matière d'éducation - et qu'il devait, de ce fait, échapper à la portée de l'article 43 de la Loi de 1982, ce dernier valant uniquement pour la modification de dispositions ne concernant qu'une ou que quelques provinces seulement (par. 110 et 111). Le juge Orsborn a carrément rejeté cet argument, au paragraphe 112 de sa décision, et a affirmé que l'article 17 des Conditions de l'union de TerreNeuve au Canada constituait une disposition en soi ou, si l'on préfère, une disposition à part (a stand alone provision). En effet, l'article 17 en question s'appliquait à la place (in lieu of) de l'article 93 de la Loi de 1867. Voir la Charte canadienne des droits et libertés, partie I de la Loi constitutionnelle de 1982, constituant l'annexe B de la Loi de 1982 sur le Canada (R.-U.), préc., note 1.

74. Arrêt Hogan 1998, préc., note 12, par. 104-105 (notre traduction). La version originale se lit comme suit:

It is obvious that, if the constitutional right is appropriately either expanded or reduced, the scope for Charter review will be correspondingly modified. However, the fact that the applicability of the Charter may be enhanced because of a constitutional amendment which removes from one province a power to discriminate does not mean that the Charter itself has been amended. 
Cette conclusion s'imposait d'elle-même selon nous. En effet, il aurait été pour le moins étonnant qu'une modification aux Conditions de l'union de Terre-Neuve au Canada soit interprétée comme constituant une modification de la Charte canadienne, et ce, bien que la portée de cette dernière se soit trouvée en pratique élargie en raison de l'abrogation des droits confessionnels garantis par lesdites conditions. Étendre la modification de ces dernières jusqu'à la Charte canadienne était un argument aberrant, que le juge Orsborn n'a d'ailleurs eu aucune difficulté à rejeter.

L'aspect fort intéressant dans la décision du juge Orsborn est qu'elle confirme l'application de la procédure sélective prévue par l'article 43 de la Loi de 1982 dans le cas précis d'une modification aux Conditions de l'union de Terre-Neuve au Canada. Plus particulièrement, le juge Orsborn a donné le feu vert à la modification en cause afin qu'elle soit accomplie de façon bilatérale, c'est-à-dire entre les autorités fédérales et Terre-Neuve. N'oublions pas cependant que ces conditions ne s'appliquaient qu'à TerreNeuve et ne concernaient que cette province, et ce, tant dans leur esprit que dans leur libellé. Il était donc normal, dans ces circonstances, d'avoir recours à l'article 43 de la Loi de 1982 et que seule la province de Terre-Neuve ait été appelée à donner son consentement à la modification souhaitée, en plus évidemment de la Chambre des communes et du Sénat.

Nous retenons néanmoins de la décision du juge Orsborn que, aux fins de l'application de l'article 43 de la Loi de 1982, il n'y a pas lieu de se préoccuper des conséquences indirectes de la modification envisagée sur le cadre constitutionnel. Seules les conséquences directes de cette dernière sur les lois constitutionnelles doivent être prises en considération.

In this case, the only proposed amendment is to Term 17 . Not a word of the Charter has been altered; the opportunity for its expanded application in Newfounland does not amount to its amendment within the contemplation of Part V of the Constitution Act, 1982. 
Dans une décision rendue le 14 janvier 1999 à l'occasion de l'arrêt Hogan, le juge Riche, de la Cour supérieure de Terre-Neuve (Newfoundland Supreme Court, Trial Division) ${ }^{75}$ a déclaré que la déconfessionnalisation du système scolaire de Terre-Neuve pouvait être accomplie en vertu de l'article 43 de la Loi de 1982 plutôt que de son article 38. Le juge Riche a déclaré par ailleurs que la liberté de conscience et de religion de même que la liberté de pensée, de croyance, d'opinion et d'expression et les droits à l'égalité n'avaient pas été enfreints en l'espèce. Il a souligné qu'aucun droit de la minorité catholique n'avait été touché dans le présent cas. Puis il a conclu de la manière suivante :

Il est, à mon avis, tout à fait clair que l'article 17 des Conditions de l'union de Terre-Neuve au Canada est une disposition constitutionnelle qui ne s'applique qu'à la province de Terre-Neuve et à aucune autre. Par conséquent, [...] la procédure bilatérale de l'article 43 est la seule procédure qui peut s'appliquer à la présente modification. Il importe peu qu'il s'agisse d'une modification de l'article 93 [de la Loi de 1867] ou de l'article 17 susmentionné. Si la modification en cause ne concerne qu'une ou que quelquesunes des provinces et non pas toutes celles-ci, alors la procédure de l'article 43 est la seule qui puisse être applicable. L'article 17 ne s'applique bien sûr qu'à TerreNeuve, et il s'ensuit que l'article 43 est la seule disposition qui puisse être utilisée pour le modifier ${ }^{76}$.

75. Le juge Riche est celui qui a entendu l'affaire au fond et qui a dû juger de la constitutionnalité de la modification constitutionnelle ayant reçu l'assentiment royal le 8 janvier 1998. Il a estimé que cette modification était constitutionnelle : voir l'arrêt Hogan 1999, préc., note 12, par. 28, 56, 71 et 75 .

76. Id., par. 69 (notre traduction). La version originale se lit comme suit : It is in my view abundantly clear that s. 17 of the Terms of Union between Newfoundland and Canada is a provision of the Constitution that applies solely to the province of Newfoundland and no other. Therefore [...] the bilateral procedure in s. 43 is the only procedure that will apply to this amendment. It does not matter whether it is an amendment to s. 93 or an amendment to Term 17. The amending procedure, if it concerns only one or more but not all provinces, then s. 43 is the only amending procedure. 
Pour rédiger sa décision, le juge Riche s'est abondamment inspiré du jugement qui avait été rendu le 16 novembre 1998 par le juge Michel Côté, de la Cour supérieure du Québec, dans l'affaire Potter CS77. Dans ce jugement, sur lequel nous reviendrons plus bas, le juge Côté avait conclu que l'adoption de la Modification constitutionnelle de 1997 (Québec) relevait de l'article 43 de la Loi de 1982 :

Ainsi, les requérants associent les mesures de protection confessionnelle en matière d'éducation, qui se retrouvent à l'article 93, aux libertés fondamentales précitées qui se retrouvent, quant à elles, à la Charte canadienne. Ils en concluent qu'une modification à l'application de l'un emporte modification implicite, bien que non explicite, de l'autre. Or, c'est précisément cette approche qu'a rejetée la Cour suprême du Canada dans l'arrêt Adler en décidant que l'article 93 de la Loi constitutionnelle de 1867 ne constitue pas une garantie de libertés fondamentales, mais qu'il constitue, en la matière dont il traite, un code complet dont la portée ne saurait être élargie par l'application de l'alinéa 2a) de la Charte canadienne. Il en va de même en l'espèce, même à rebours. Le retrait - à l'égard de la seule province de Québec - d'une restriction constitutionnelle à la plénitude de sa compétence de légiférer en matière d'éducation, ne peut avoir pour effet de modifier la liberté fondamentale de chacun relative à la religion que garantit l'alinéa $2 a$ ) de la Charte canadienne, pas plus qu'il n'affecte son égalité de bénéfice et protection égale de la loi qu'accorde l'article 15 de cette même charte ${ }^{78}$.

Enfin, toujours dans l'arrêt Hogan, la Cour d'appel de TerreNeuve (Newfoundland Supreme Court, Court of Appeal) a rendu sa décision le 28 février $2000^{79}$. Au nom de cette cour, le juge Cameron a alors confirmé la décision du juge Riche de la Cour suprême de Terre-Neuve, voulant que la modification accomplie en 1998

Term 17, of course, applies only to Newfoundland and it follows that s. 43 is the provision that must be used to amend it.

77. $\quad$ Arrêt Potter CS, préc., note 14.

78. Id., par. 48.

79. Arrêt Hogan $C A$, préc., note 12. 
concernant la déconfessionnalisation du système scolaire terreneuvien ait été valide ${ }^{80}$. Selon la Cour d'appel, l'article 43 de la Loi de 1982 était la disposition appropriée pour accomplir pareille modification :

La fausse prémisse sur laquelle se fondent ces arguments veut que la Constitution fournisse un standard commun et national de protection en faveur de l'instruction confessionnelle. Le Canada n'est pas un État unitaire. La Confédération, dans sa nature même, reconnaît qu'il puisse y avoir des différences d'une province à l'autre.

Bien sûr, ce que l'article 93 a fait pour les provinces auxquelles il s'applique a été de protéger le système ou les systèmes d'éducation en vigueur dans chacune de ces provinces au moment de leur entrée dans la Confédération. Il n'a pas établi un standard national. Il était plutôt rédigé de façon à ne pas établir pareil standard. Au Québec, ainsi qu'il appert des références que nous avons faites ci-dessus à la situation qui régnait dans cette province, il existait plusieurs systèmes, selon l'endroit où l'on vivait. Dans d'autres provinces (Nouvelle-Écosse et NouveauBrunswick), il n'y avait, au moment de la naissance de la Confédération, qu'un système public d'éducation: par conséquent, les protections en matière d'écoles confessionnelles que contenait l'article 93 n'avaient pas vraiment d'application dans ces provinces. Dans le contexte propre à ce pays, l'éducation est considérée comme étant de compétence provinciale et les différences sont valorisées. Il n'y a dès lors rien en principe qui empêche une application différenciée de la Charte dans les diverses provinces en matière d'éducation, parce que les protections offertes par l'article 93 (comme celle qui découle de l'article 17) sont différentes pour chaque province.

L'idée voulant que la modification de l'article 17 soit une modification de la Charte parce que l'activité visée sera, à l'avenir, couverte par la Charte ne résiste pas à une analyse

80. Il s'agissait de la modification dont nous avons parlé plus haut : voir les notes 72 et 75 . 
fouillée. Chaque fois qu'une législature adopte une loi, il y a un risque qu'une activité qui n'était jusqu'alors pas couverte par la Charte le devienne. Personne ne peut qualifier de telles modifications à la Charte de "modifications constitutionnelles". En l'espèce, l'activité qui, en vertu de l'article 17, a été mise à l'abri de la Charte dans le passé fait maintenant l'objet de l'application de celle-ci. En cela, cependant, la modification envisagée n'est en rien différente de n'importe quelle loi adoptée par une législature ou le Parlement. Aucun principe, aucun mot ni aucune nuance contenu dans la Charte n'est changé du fait de la modification de l'article 17. Cette dernière n'est pas une modification de la Charte et le choix de la procédure applicable à cette modification ne doit pas être effectué sur cette base ${ }^{81}$.

81. Arrêt Hogan CA, préc., note 12, par. 93-95 (notre traduction). La version originale se lit comme suit :

The false premise which is at the foundation of these arguments is that the Constitution provides some common national standard of protection for denominational education. Canada is not a unitary state. Confederation, by its very nature, recognizes there will be differences in laws from province to province. [...]

Of course, what s. 93 did for the provinces to which it applies was to protect the system or systems of education in effect in each of those provinces at the time they entered Confederation. This did not establish a national standard. It was in fact designed not to establish a national standard. In Quebec, as the earlier references to the situation in that province indicate, there were different systems depending on where one lived. In some provinces (Nova Scotia and New Brunswick) there was, at Confederation, only a public education system and therefore the denominational education protections contained in s. 93 have no real application in those provinces. In the context of this country, education is considered to be a provincial matter and differences are celebrated. There is, therefore, nothing in principle to prevent the different application of the Charter in different provinces to matters related to education because the protections in s. 93 (or provisions such as Term 17) are different for different provinces. The idea that the amendment to Term 17 must be an amendment to the Charter because activity which hitherto was not subject to Charter scrutiny will, in the future, be subject to the Charter does not survive reasoned examination. Every time a legislature passes a statute there is the potential for Charter scrutiny of activity 
La Cour d'appel de Terre-Neuve s'est donc limitée à l'examen de la disposition modifiée et du caractère technique de la modification envisagée 82 . Elle a par ailleurs rappelé que la procédure sélective de l'article 43 de la Loi de 1982 ne pouvait pas être mise en œuvre facilement, car elle requerrait non seulement le consentement de la province visée mais aussi celui des autorités fédérales, ces dernières veillant en tout état de cause à ce que les droits des minorités soient protégés ${ }^{83}$.

\section{J) Le Renvoi relatif à la sécession du Québec}

Dans le Renvoi relatif à la sécession du Québec, la Cour suprême a émis quelques commentaires sur la portée de l'expression "Constitution du Canada ", qui figure aux articles 38, 41, 42, 43 et 44 de la Loi de 1982 :

Comme nous l'avons confirmé dans le Renvoi: Opposition du Québec à une résolution pour modifier la Constitution, [1982] 2 R.C.S. 793, à la p. 806 : " La Loi constitutionnelle de 1982 est maintenant en vigueur. Sa légalité n'est ni contestée ni contestable. "La "Constitution du Canada" comprend certainement les textes énumérés au par. 52(2) de la Loi constitutionnelle de 1982. Même si ces textes jouent un rôle de premier ordre dans la détermination des règles constitutionnelles, ils ne sont pas exhaustifs. La Constitution "comprend des règles non écrites - et écrites - ", comme nous l'avons souligné

82.

which was not subject to scrutiny before. No one would describe those changes as amendments to the Charter requiring constitutional process for amendment. Here, activity which may, by virtue of Term 17, have been exempted from the application of the Charter in the past, now becomes open to Charter scrutiny. In this, however, the amendment would be no different than many new statutes passed by a Legislature or Parliament. Not a principle, word or nuance of the Charter itself is changed by an amendment to Term 17. The amendment to Term 17 is not an amendment to the Charter and the choice of the procedure for amendment of Term 17 cannot be determined on that basis.

83. Id., par. 97 et 125 . 
récemment dans le Renvoi relatif aux juges de la Cour provinciale, précité, au par. 92. Enfin, selon le Renvoi relatif au rapatriement, précité, à la p. 874 , la Constitution du Canada comprend le système global des règles et principes qui régissent la répartition ou l'exercice des pouvoirs constitutionnels dans l'ensemble et dans chaque partie de l’État canadien.

Ces règles et principes de base, qui comprennent les conventions constitutionnelles et les rouages du Parlement, font nécessairement partie de notre Constitution, parce qu'il peut survenir des problèmes ou des situations qui ne sont pas expressément prévus dans le texte de la Constitution. Pour résister au passage du temps, une constitution doit comporter un ensemble complet de règles et de principes offrant un cadre juridique exhaustif pour notre système de gouvernement. Ces règles et principes ressortent de la compréhension du texte constitutionnel lui-même, de son contexte historique et des diverses interprétations données par les tribunaux en matière constitutionnelle ${ }^{84}$.

Il en résultait donc nettement que, pour la Cour suprême, l'article 52 (2) de la Loi de 1982 - qui offre, ne l'oublions pas, une définition de la Constitution du Canada - n'était pas limitatif et que la Constitution canadienne comprenait tant des règles non écrites que des règles écrites. C'est sur cette base que la Cour suprême a énoncé quatre principes directeurs fondamentaux qui, selon elle, sont sous-jacents à l'architecture constitutionnelle canadienne : le fédéralisme, la démocratie, le constitutionnalisme et la primauté du droit ainsi que le respect des minorités ${ }^{85}$.

Déjà, dans le Renvoi: Droits linguistiques au Manitoba ${ }^{86}$, la Cour suprême avait eu l'occasion de dire que la primauté du droit

84. Renvoi relatif à la sécession du Québec, préc., note 13, par. 32.

85. Id. Notons que la Cour suprême a précisé que cette énumération des principes constitutionnels sous-jacents n'était pas exhaustive.

86. Renvoi : Droits linguistiques au Manitoba, [1985] 1 R.C.S. 721. 
était un "principe [...] nettement implicite de par la nature même d'une constitution 87 ".

Dans l'arrêt Fraser c. Commission des relations de travail dans la Fonction publique 88 , la Cour suprême avait dit de la liberté de parole qu'elle constituait " une valeur profondément enracinée dans notre système de gouvernement démocratique 89 ". À ses yeux, " [i]1 s'agit d'un principe de notre constitution de common law, que nous avons hérité du Royaume-Uni en vertu du préambule de la Loi constitutionnelle de 186790 ". La Cour suprême a ajouté ce qui suit :

Toutefois, il ne s'agit pas d'une valeur absolue. Il n'y a probablement aucune valeur absolue. Toutes les valeurs importantes doivent être restreintes et évaluées en fonction d'autres valeurs importantes et souvent concurrentes. Ce processus de définition, de restriction et d'évaluation est aussi nécessaire pour ce qui est de la "liberté de parole " en tant que valeur qu'elle l'est pour les autres valeurs ${ }^{91}$.

De même, dans l'arrêt La Reine c. Beauregard92, la Cour suprême avait eu l'occasion de dire que le principe de l'indépendance judiciaire avait été transféré du Royaume-Uni au Canada par le texte du préambule de la Loi de $1867^{93}$.

87. Id., 750 .

88. Fraser c. Commission des relations de travail dans la Fonction publique, [1985] 2 R.C.S. 455.

89. Id., par. 20.

90. Id.

91. Id., par. 21.

92. $\quad$ La Reine c. Beauregard, [1986] 2 R.C.S. 56.

93. Id.; voir particulièrement au paragraphe 29 : "Étant donné que l'indépendance judiciaire est depuis des siècles un principe important de la Constitution du Royaume-Uni, on peut à juste titre déduire que ce principe a été transféré au Canada par le texte constitutionnel du préambule ". Au paragraphe 30, voici comment la Cour s'est exprimée :

En résumé, l'histoire de la Constitution du Canada et le droit constitutionnel canadien actuel établissent clairement les racines profondes, la vitalité et le caractère vibrant contemporains du principe de l'indépendance judiciaire au Canada. Le rôle des tribunaux en tant qu'arbitres des litiges, interprètes du droit et défenseurs de la Constitution exige qu'ils soient complètement 
Il découle donc du Renvoi relatif à la sécession du Québec, et des autres décisions de la Cour suprême dont nous venons de parler, que l'expression "Constitution du Canada " dans l'article 52 (2) de la Loi de 1982 est susceptible d'inclure, outre les documents expressément mentionnés dans cette dernière mesure, un certain nombre de principes constitutionnels dont la portée et l'effet normatif réels demeurent toutefois énigmatiques.

\section{K) L'arrêt Potter}

Dans l'arrêt Potter, la validité d'une modification constitutionnelle pour permettre la déconfessionnalisation du système scolaire québécois était contestée ${ }^{94}$. La Cour d'appel du Québec a conclu, sous la plume du juge Baudouin (au nom de ses collègues Chamberland et Biron), que cette modification bilatérale Ottawa-Québec avait été valablement accomplie en vertu de l'article 43 de la Loi de 1982. Notons au passage que la modification en question avait été sanctionnée par le gouverneur général du Canada

séparés, sur le plan des pouvoirs et des fonctions, de tous les autres participants au système judiciaire.

Notons que, dans l'arrêt La Reine c. Beauregard, préc., note 92, les juges majoritaires de la Cour suprême ont également rappelé que, outre le principe de l'indépendance judiciaire, deux autres principes formaient la pierre angulaire du système judiciaire britannique : la suprématie du Parlement et la primauté du droit (par. 25). En ce qui touche plus particulièrement l'indépendance judiciaire, la Cour suprême a précisé, outre le préambule de la Loi de 1867, deux autres sources ou raisons d'être : le fédéralisme et la Charte canadienne (par. 27-28). Voir aussi le Renvoi relatif aux juges de la Cour provinciale, préc., note 11, où la Cour suprême a réitéré que le principe de l'indépendance judiciaire avait été incorporé dans la Constitution canadienne, en tant que norme non écrite, par l'entremise du préambule de la Loi de 1867. Toujours dans ce dernier renvoi, la Cour suprême avait statué que le préambule en question invitait " les tribunaux à transformer [les principes structurels de la Loi de 1867] en prémisses d'une thèse constitutionnelle qui am[enait] à combler les vides des dispositions expresses du texte constitutionnel " (par. 104).

94. Modification constitutionnelle de 1997 (Québec), TR/1997-141 (Gaz. Can. II), édition spéciale. Par cette modification, l'article 93A a été ajouté à la Loi de 1867. Cet article prévoit que les paragraphes 1 à 4 de l'article 93 de cette loi, portant sur les droits confessionnels, ne s'appliquent pas au Québec. 
le 19 décembre 1997 et que la décision de la Cour d'appel du Québec datait du 12 novembre 2001, ce qui rendait à peu près impensable toute autre conclusion que celle qui allait dans le sens de la validité de cette modification.

Quoi qu'il en soit, toujours dans l'arrêt Potter, le juge Baudouin a aisément rejeté l'argument voulant que la modification bilatérale susmentionnée ait eu un impact sur le partage des compétences législatives en matière d'éducation ${ }^{95}$. Du reste, pour les mêmes motifs que ceux qui avaient été invoqués par les juges Orsborn et Riche ainsi que par la Cour d'appel de Terre-Neuve dans l'arrêt Hogan - motifs dont nous avons parlé ci-dessus -, le juge Baudouin a déclaré que ladite modification bilatérale n'avait pas eu pour effet de modifier l'article 29 de la Charte canadienne ${ }^{96}$. Cela était d'une grande évidence.

Le juge Baudouin s'est toutefois penché sur une question beaucoup plus délicate, soit celle de savoir si l'article 43 de la Loi de 1982 requerrait le consentement d'autres provinces que le Québec $^{97}$. Pour comprendre cette question, il faut savoir que l'article 93 de la Loi de 1867 s'applique en ce moment à cinq provinces, soit 1'Ontario, la Nouvelle-Écosse, le NouveauBrunswick, la Colombie-Britannique et l'Île-du-Prince-Édouard ${ }^{98}$. Il s'appliquait aussi au Québec avant que celui-ci s'en soustraie par la modification bilatérale de 1997. Malgré tout, le juge Baudouin en

95. Arrêt Potter CA, préc., note 14, par. 20-27.

96. Id., par. 28-32. Plus spécialement, le juge Baudouin en est venu à la conclusion que la Charte canadienne ne confère en elle-même aucune garantie quant aux droits confessionnels et que ceux-ci sont, au contraire, des exceptions à la Charte canadienne.

97. Id., par. 33-52.

98. Les lois constitutives du Manitoba, de l'Alberta et de la Saskatchewan contiennent en ce moment des dispositions qui sont analogues à celles de l'article 93 de la Loi de 1867, mais qui comportent néanmoins certaines différences. C'était aussi le cas pour la province de Terre-Neuve avant qu'elle déconfessionnalise son système scolaire au moyen d'une modification de l'article 17 des Conditions de l'union de Terre-Neuve au Canada, modification dont nous avons parlé plus haut, notamment aux notes 72 et 75 . 
est venu à la conclusion que seul le Québec, parmi toutes les provinces auxquelles l'article 93 s'appliquait, devait donner son consentement aux termes de l'article 43 de la Loi de 1982, en plus évidemment de la Chambre des communes et du Sénat ${ }^{99}$.

Plus exactement, le juge Baudouin s'est fondé sur la version anglaise de l'article 43 qui parle du consentement de chaque province à laquelle la modification s'applique (" of each province to which the amendment applies "). Le juge Baudouin a également examiné la version française de l'article 43, qui requiert le consentement " de chaque province concernée " par la modification envisagée. Il en a conclu que le verbe concerner signifiait, dans son sens courant, "s'appliquer à " ou "avoir rapport à ", ce qui correspondait, selon lui, à la version anglaise où l'on trouvait le verbe applies ${ }^{100}$.

Or, pour le juge Baudouin, il était clair que la modification de 1997 ne s'appliquait techniquement qu'au Québec et que seule cette province devait consentir à cette modification (en plus des autorités fédérales) aux fins de l'application de l'article 43 de la Loi de 1982. En d'autres termes, le juge Baudouin a considéré que seul le Québec était " concerné " par la modification de 1997, et ce, en dépit du fait que l'article 93 de la Loi de 1867 avait touché cinq autres provinces à toute époque pertinente.

99. Arrêt Potter $C A$, préc., note 14. Notons cependant que le Sénat ne dispose que d'un veto suspensif de 180 jours en vertu de l'article 47 de la Loi de 1982.

100. Id., par. 46. Selon le juge Baudouin, la version anglaise de la Loi de 1982 " correspond[ait] très exactement à l'intention des autorités constituantes " (id., par. 49). Ces dernières ont voulu donner à l'ensemble du processus de modification constitutionnelle "une certaine souplesse, tout en l'entourant d'un contrôle démocratique de garanties juridiques et politiques importantes ". Très nettement, sur la question de l'équivalence entre les versions française et anglaise de l'article 43 de la Loi de 1982, le juge Baudouin a accordé beaucoup de poids à un article du professeur Daniel Proulx au paragraphe 46 de son jugement: Daniel ProulX, "La modification constitutionnelle de 1997 relative aux structures scolaires au Québec : une mesure opportune et juridiquement solide ", (1998) 58 R. $d u$ B. 41. 
Il ressort donc de cette décision de la Cour d'appel du Québec que, contrairement à l'article 45 de la Loi de 1982, qui doit se voir donner une interprétation téléologique, l'article 43 de cette loi, quant à lui, doit être interprété de façon technique101. Et cela s'avère, semble-t-il, même si ce dernier article doit être interprété en tenant compte de l'article 45 en question ${ }^{102}$.

\section{L) Le Renvoi relatif à la Loi sur la Cour suprême}

Dans le Renvoi relatif à la Loi sur la Cour suprême, cette cour a reconnu que les changements à sa composition ${ }^{103}$ et son abolition dépendaient de l'article 41 d) de la Loi de 1982. Elle a aussi conclu que les changements à ses autres caractéristiques essentielles ${ }^{104}$ relevaient de la procédure $7 / 50$, en vertu de l'article 42 (1)d) de cette

101. On peut dire de la procédure sélective de l'article 43 de la Loi de 1982 qu'elle requiert un consentement provincial qui, dans certains cas, peut être relativement limité, puisqu'elle ne repose que sur le consentement de la ou des provinces directement visées par la modification constitutionnelle envisagée (en plus de celui des autorités fédérales). Cela ne peut toutefois qu'encourager le fédéralisme coopératif, c'est-à-dire la collaboration fédérale-provinciale (ou fédérale-provinciale-territoriale).

102. Il est clair que l'article 43 de la Loi de 1982 doit être interprété à la lumière de l'article 45 de cette loi. Ainsi, l'article 43 vise essentiellement des dispositions constitutionnelles qui ne concernent pas que la régie interne d'une seule province mais qui, néanmoins, ne s'appliquent qu'à une ou à quelques provinces seulement, et non à toutes celles-ci. Parmi les mesures visées par l'article 43 en question figurent, au premier chef, les dispositions constitutionnelles qui (1) transcendent le seul intérêt d'une province, (2) ne s'appliquent qu'à une ou à quelques provinces et (3) mettent en cause des intérêts fédéraux. Reste maintenant à déterminer de quelles dispositions il s'agit...

103. La Cour suprême a précisé, dans le Renvoi relatif à la Loi sur la Cour suprême, préc., note 15, que la notion de "composition " de la Cour suprême renvoyait à l'article 4 (1) de même qu'aux articles 5 et 6 de la Loi sur la Cour suprême, préc., note 67. Selon la Cour suprême, ces dispositions codifient sa composition et les conditions de nomination de ses juges telles qu'elles existaient en 1982.

104. La Cour suprême a dit dans le Renvoi relatif à la Loi sur la Cour suprême, préc., note 15, par. 94, que les " caractéristiques essentielles incluent, à tout le moins, la juridiction de la Cour en tant que cour générale d'appel de dernier ressort pour le Canada, notamment en matière d'interprétation de la Constitution, et son indépendance". 
loi. Voici d'ailleurs comment la Cour suprême s'est exprimée à ce sujet :

Initialement, la Cour suprême a acquis son statut constitutionnel en raison de l'évolution historique qui en a fait une institution dont la pérennité et le fonctionnement affectaient les intérêts à la fois du Parlement et des provinces. Ce statut a ensuite été confirmé dans la Loi constitutionnelle de 1982, dont le contenu reflète la perception que les caractéristiques essentielles de la Cour faisaient partie de la Constitution canadienne.

$[\ldots]$

En conséquence, la Loi constitutionnelle de 1982 a confirmé que les caractéristiques essentielles de la Cour suprême sont protégées par la Constitution. En effet, la partie V de la Loi constitutionnelle de 1982 assujettit expressément les changements touchant la Cour suprême et sa composition au respect des procédures de modification de la Constitution.

$[\ldots]$

En résumé, la Cour suprême a acquis son statut constitutionnel parce qu'elle a évolué de manière à devenir une cour générale d'appel de dernier ressort pour le Canada, avec compétence sur les appels relatifs à toutes les lois fédérales et provinciales, y compris la Constitution. Ce statut a été confirmé dans la Loi constitutionnelle de 1982, qui a assujetti toute modification portant sur la composition de la Cour et ses autres caractéristiques essentielles à des procédures de modification strictes ${ }^{105}$.

Notons que ces conclusions n'étaient pas évidentes, puisque la Loi sur la Cour suprême ne figure pas parmi les textes mentionnés à l'article 52 (2) de la Loi de 1982. Il y avait donc lieu de s'interroger sur la question de savoir si la modification des dispositions de la Loi sur la Cour suprême était ou non protégée par la partie V de la Loi

105. Id., par. 76,90 et 95 . 
de 1982. Or, la Cour suprême a carrément rejeté l'argument du procureur général du Canada, voulant qu'elle-même ne soit pas visée par la procédure de modification constitutionnelle de la partie V de la Loi de 1982, parce que la Loi sur la Cour suprême n'est pas mentionnée à l'article 52 de cette loi. La Cour suprême a dit ce qui suit :

Cet argument est dépourvu de fondement. Il signifierait que les auteurs du texte constitutionnel ont constitutionnalisé l'exclusion de la Cour suprême de toute protection constitutionnelle [...] Cet argument signifierait aussi que les provinces ont accepté de mettre ce pouvoir unilatéral du Parlement à l'abri de toute modification, à moins que ne soient respectées les procédures astreignantes établies dans la partie $\mathrm{V}^{106}$.

La Cour suprême a aussi rejeté du revers de la main l'autre argument avancé par le procureur général du Canada, voulant que l'accord du lac Meech et l'entente de Charlottetown auraient, s'ils avaient été adoptés, constitutionnalisé les conditions de nomination à la Cour suprême mais que, puisqu'ils ont finalement échoué, lesdites conditions ne soient pas inscrites dans la Constitution. La Cour suprême a répondu à cet argument de la façon suivante :

Nous ne pouvons pas retenir cet argument. Comme nous l'avons rappelé, l'adoption de la Loi constitutionnelle de 1982 a protégé le statu quo en ce qui concerne la Cour suprême. Ce statu quo incluait expressément la composition de la Cour, dont la représentation du Québec à la Cour fait partie intégrante. L'Accord du lac Meech et l'Accord de Charlottetown auraient réformé le processus de

106. Id., par. 98. Notons que la Cour suprême a affirmé que ses propres caractéristiques essentielles constituaient un élément fondamental de l'architecture constitutionnelle canadienne et que c'était à ce titre qu'elles se trouvaient enchâssées dans la Constitution du pays (par. 100-101). La Cour suprême a même invoqué l'article 101 de la Loi de 1867 au soutien de son opinion et a conclu que cet article " exige maintenant que le Parlement préserve - et protège - les éléments essentiels qui permettent à la Cour suprême de s'acquitter de sa mission actuelle " (par. 101). 
nomination des juges de la Cour et exigé que les juges pour le Québec soient nommés parmi les candidats figurant sur une liste soumise par le Québec. Ces vaines tentatives de réforme prouvent uniquement qu'on a tenté de réformer plus généralement le processus de sélection, mais ne nous apprennent rien sur la protection constitutionnelle actuelle de la Cour. L'échec de l'Accord du lac Meech et de l'Accord de Charlottetown signifie simplement que le statu quo quant au rôle constitutionnel de la Cour est demeuré intact ${ }^{107}$.

De façon générale, il ressort du Renvoi relatif à la Loi sur la Cour suprême que cette dernière jouit d'un statut constitutionnel du moins quant à ses caractéristiques essentielles - en raison de son rôle primordial dans la structure même du Canada ${ }^{108}$. La Cour suprême forme " une institution constitutionnellement essentielle qui affecte les intérêts à la fois du fédéral et des provinces ${ }^{109}$ "; elle est " une pierre d'assise de la Constitution"10".

\section{M) Le Renvoi relatif à la réforme du Sénat}

Dans le Renvoi relatif à la réforme du Sénat, la Cour suprême s'est penchée sur la portée des modalités de modification constitutionnelle énoncées dans la partie V de la Loi de 1982 et sur leur dynamique intrinsèque.

La Cour suprême devait alors essentiellement se prononcer sur la possibilité pour le Parlement de modifier unilatéralement la Constitution canadienne en vertu de l'article 44 de la Loi de 1982, de façon à tenir des élections facultatives en vue de nommer des sénateurs ou de réduire la durée de leur mandat. D'autres questions, portant sur l'abolition du Sénat ou sur l'abrogation des

107. Id., par. 103.

108. À cet égard, voir particulièrement id., par. 87, 88 et 100 .

109. Id., par. 87.

110. Id., par. 89. 
paragraphes 3 et 4 de l'article 23 de la Loi de $1867^{111}$, étaient aussi posées à la Cour suprême, par la même occasion, par les autorités fédérales.

La Cour suprême a conclu que le Parlement ne pouvait pas apporter unilatéralement au Sénat les changements proposés quant à la tenue d'élections sénatoriales ni quant à la durée du mandat des sénateurs. Selon la Cour suprême, de tels changements exigeaient l'application de la procédure $7 / 50$, et ce, que ce soit en vertu de l'article 42 de la Loi de 1982 dans le cas de l'élection ou de son article 38 concernant le mandat112. Pour l'abolition du Sénat,

111. Le paragraphe 3 de l'article 23 de la Loi de 1867 concerne l'avoir foncier, alors que le paragraphe 4 porte sur l'avoir net ou, en d'autres mots, la fortune personnelle des sénateurs.

112. Comme devait l'affirmer la Cour suprême dans le Renvoi relatif à la réforme du Sénat, préc., note 16, par. 74, 75 et 82 :

Nous convenons que le texte de l'art. 42 ne mentionne pas les changements à la durée du mandat des sénateurs. Cela ne veut pas dire pour autant que la procédure de modification unilatérale par le Parlement prévue à l'art. 44 s'applique à tous les changements relatifs au Sénat qui ne sont pas visés par l'art. 42: voir Whyte, p. 102-103.

Nous ne pouvons souscrire à l'interprétation que propose le procureur général du Canada de la portée de l'art. 44. Nous avons déjà indiqué que la procédure de modification unilatérale par le Parlement a une portée restreinte. Il ne s'agit pas d'une procédure dont le champ d'application est vaste et qui vise les changements constitutionnels à tous les aspects du Sénat que ne vise pas expressément une autre procédure de modification décrite à la partie V. L'historique, les termes et la structure de la partie V indiquent que la procédure normale de modification de la Constitution est prévue à l'art. 38 plutôt qu'à l'art. 44. Les changements qui mettent en cause les intérêts des provinces relatifs au Sénat en tant qu'institution faisant partie intégrante du système fédéral ne peuvent être apportés qu'en application de la procédure normale de modification. L'article 44, qui constitue une exception à la procédure normale, envisage des mesures prises en vue du maintien et du changement du Sénat, sans pour autant modifier ses nature et rôle fondamentaux. [...]

L'imposition d'un mandat fixe, si long soit-il, constitue un changement qui engage les intérêts des provinces en tant que parties prenantes dans l'ordre constitutionnel canadien et exige 
la Cour suprême a retenu la procédure de l'unanimité qui figure dans l'article 41 de la Loi de 1982.

Enfin, la Cour suprême a accepté que la procédure unilatérale fédérale de l'article 44 s'applique à l'abrogation du paragraphe 3 de l'article 23 de la Loi de 1867. Elle en est venue à la même conclusion en ce qui concerne le paragraphe 4, sauf en ce qui avait trait aux sénateurs venant du Québec. Dans ce dernier cas, puisque cette province prenait part à un arrangement particulier, la Cour suprême a appliqué l'article 43 de la Loi de 1982 et a requis le consentement des autorités fédérales et de la province précisément visée : le Québec ${ }^{113}$.

Pour parvenir à ces conclusions, la Cour suprême s'est abondamment inspirée de la nature et de l'histoire du Sénat, comme elle l'avait fait antérieurement dans le Renvoi relatif à la Chambre haute, dont nous avons parlé ci-dessus.

Plus particulièrement, sur les modalités de modification constitutionnelle en tant que telles, la Cour suprême a affirmé ce qui suit :

- L'article 52 (2) de la Loi de 1982 ne donne pas une définition exhaustive de la Constitution du Canada114. Au contraire,

l'application de la procédure normale - celle du 7/50 énoncée à l'art. 38 - pour que se réalise cette modification constitutionnelle. Cela revient à dire que les conditions essentielles du Sénat qui ne sont pas visées par l'article 42 de la Loi de 1982 le sont plutôt par l'article 38 de cette loi.

113. Plus précisément, la Cour suprême a été d'avis que la suppression de la condition relative à l'avoir foncier, prévue dans l'article 23 (3) de la Loi de 1867, ne modifierait ni la nature du Sénat ni son rôle fondamental. Cependant, la suppression de cette condition pour les sénateurs du Québec constituerait une modification relative à un arrangement spécial et entraînerait l'application de l'article 43 de la Loi de 1982 : Renvoi relatif à la réforme du Sénat, id., par. 91.

114. Sur le caractère non exhaustif de l'article 52 (2) de la Loi de 1982, voir id. au par. 24. Notons d'ailleurs que, dans le Renvoi relatif à la réforme du Sénat, la Cour suprême a rappelé qu'elle avait aussi mentionné le caractère non exhaustif de l'article 52 (2) dans (au moins) deux de ses 
cette dernière inclut aussi les différents principes qui sont sous-jacents aux textes constitutionnels, soit les prémisses qui façonnent la structure fondamentale du pays. Ceux-ci sont donc compris dans les modalités de modification constitutionnelle de la partie V de la Loi de 1982, tout comme d'ailleurs les textes mêmes qui composent la Constitution canadienne ${ }^{115}$;

- Les articles 44 et 45 de la Loi de 1982 mettent respectivement en cause des intérêts purement fédéraux ou provinciaux ${ }^{116}$;

- Les modifications constitutionnelles qui touchent à des intérêts provinciaux doivent échapper à la portée de l'article 44 de la Loi de $1982^{117}$;

- L'article 42 de la Loi de 1982 complète l'article 38 de cette dernière ${ }^{118}$. Il poursuit deux buts différents : 1) assurer que la procédure $7 / 50$ s'applique aux sujets qu'il mentionne expressément; 2) déclarer que les provinces ne peuvent pas se soustraire aux modifications accomplies sous son égide ${ }^{119}$;

décisions antérieures, soit le Renvoi relatif à la sécession du Québec, préc., note 13, par. 32, et le Renvoi relatif à la Loi sur la Cour suprême, préc., note 15, par. 97-100.

115. Sur ce point, voir notamment le Renvoi relatif à la réforme du Sénat, préc., note 16, par. 27, où la Cour suprême affirme ce qui suit :

[La] Constitution ne doit pas être considérée comme un simple ensemble de dispositions écrites isolées. Elle a une architecture, une structure fondamentale. Par extension, les modifications constitutionnelles ne se limitent pas aux modifications apportées au texte de la Constitution. Elles comprennent aussi les modifications à son architecture.

116. Id., par. 32 .

117. Id., par. 34.

118. Id., par. 37.

119. Sur ce point, voir id., par. 38 :

Cette disposition poursuit deux objectifs. Premièrement, donner aux auteurs de la Loi constitutionnelle de 1982 la certitude que la procédure $7 / 50$ s'applique aux modifications portant sur certaines questions qui y sont expressément incluses : J. D. Whyte, "Senate Reform: What Does the Constitution Say? ", dans J. Smith, dir., The Democratic Dilemma: Reforming the 
- L'article 43 de la Loi de 1982 entre en jeu lorsqu'on cherche à modifier des dispositions de la Constitution du Canada qui prévoient un arrangement spécial pour une ou quelques provinces. Il requiert alors le consentement de chacune des provinces en question ${ }^{120}$.

Notons du reste que, dans le Renvoi relatif à la réforme du Sénat, la Cour suprême n'a pas écarté complètement la possibilité que la doctrine du caractère véritable s'applique en matière de modification constitutionnelle. Elle s'est tout simplement contentée de dire que cette doctrine, si elle devait effectivement être prise en

Canadian Senate (2009), 97, p. 102. Deuxièmement, déclarer que le droit des provinces de " se soustraire " à certaines modifications visées aux par. 38(2) à (4) ne s'applique pas aux catégories de modifications prévues à l'art. 42. Ainsi, les modifications apportées sous le régime de l'art. 42 s'appliquent de manière uniforme à toutes les provinces et les changements visés par la disposition sont mis en œuvre de manière cohérente partout au pays.

Voir aussi ce que dit la Cour suprême au paragraphe 83 :

Fait à noter, bien que l'art. 42 ne s'applique pas à l'imposition d'un mandat d'une durée fixe, aucune province ne pourrait "se soustraire " à une réforme du mandat qui recueillerait le consentement requis des provinces. Un tel changement constituerait une réforme institutionnelle qui modifierait l'indépendance du Sénat et le poste de sénateur. Cependant, il ne toucherait pas la compétence législative, les droits de propriété ou tous autres droits ou privilèges d'une législature ou d'un gouvernement provincial. En conséquence, il ne ferait pas intervenir le droit de "se soustraire " prévu au par. 38(2) de la Loi constitutionnelle de 1982.

120. Voir id., par. 44 :

L'art. 43 entre en jeu, à tout le moins, lorsqu'une modification constitutionnelle touche une disposition de la Constitution du Canada qui prévoit un "arrangement spécial", applicable uniquement à une ou à certaines des provinces. En pareil cas, le recours à la procédure 7/50 aurait une portée trop large, car il subordonnerait l'adoption de la modification au consentement de provinces auxquelles ne s'applique pas la disposition. L'article 43 permet aussi d'éviter qu'une disposition qui prévoit un arrangement spécial puisse être modifiée sans le consentement des provinces visées par l'arrangement: Monahan et Shaw, p. 210 . 
considération, ne saurait justifier une interprétation stricte des termes mêmes de la partie V de la Loi de $1982^{121}$.

Enfin, en ce qui concerne l'abolition du Sénat, la Cour suprême a dit que celle-ci exigerait l'application de la règle de l'unanimité, étant donné qu'elle " modifierait de façon fondamentale la structure constitutionnelle canadienne, y compris la procédure

121. Sur cette question, voir id., par. 66-67. La Cour suprême y affirme ce qui suit :

Dans un même ordre d'idée, le procureur général du Canada plaide que des élections consultatives ne constitueraient pas, " de par leur caractère véritable", des modifications portant sur le " mode de sélection des sénateurs ". Il s'appuie ici sur la doctrine du caractère véritable utilisée par les tribunaux dans les affaires de partage des compétences pour décider si une loi a été validement adoptée par un ordre de gouvernement : voir Hogg, p. 15-7 à 15-10. Les tribunaux se demandent dans de tels cas si la loi, de par son objet et ses effets, relève d'un des chefs de compétence constitutionnelle de l'autorité législative : Renvoi relatif à la Loi sur les valeurs mobilières, 2011 CSC 66, par. 63-66. Selon le procureur général, une loi visant la mise en place d'élections consultatives ne constituerait pas, de par son objet et ses effets, une modification relative au "mode de sélection des sénateurs". Il restreint ainsi le sens de cette expression au mécanisme officiel de nomination des sénateurs par le gouverneur général.

Comme nous l'avons vu, l'expression "mode de sélection des sénateurs", suivant le sens ordinaire des termes qui le composent, ne vise pas uniquement le mécanisme officiel de nomination. L'emprunt d'une doctrine issue de la jurisprudence sur le partage des compétences n'écarte pas les difficultés que pose le texte constitutionnel. Même si la doctrine du caractère véritable était pertinente pour la présente analyse, son application ne saurait justifier une interprétation restrictive des dispositions pertinentes relatives à la modification de la Constitution. En effet, il convient de rappeler que les art. 38 et 42 de la Loi constitutionnelle de 1982 visent à assurer qu'un consentement provincial appréciable sera obtenu pour les modifications constitutionnelles mettant en cause des intérêts provinciaux. La procédure 7/50 constitue la règle générale applicable pour modifier la Constitution canadienne. Le fait pour le procureur général d'invoquer le " caractère véritable " ne modifie en rien ce principe. 
de modification de la Constitution 122 ". Selon la Cour suprême, le fait de dépouiller totalement le Sénat de ses pouvoirs et de réduire à zéro le nombre de sénateurs outrepasserait la portée de l'article 42 de la Loi de 1982123. Et la Cour suprême d'ajouter que la suppression de la Chambre haute transformerait fondamentalement la structure et l'application de la partie V de la Loi de 1982 dans son ensemble, et serait protégée par l'article $41 e$ ) de cette loi ${ }^{124}$. La Cour suprême a conclu ainsi :

L'argument du procureur général du Canada voulant qu'on puisse supprimer la chambre haute sans modifier la partie $\mathrm{V}$ ne nous convainc pas. Comme nous l'avons mentionné précédemment, la notion de modification de la Constitution du Canada ne vise pas seulement les modifications de forme; elle englobe aussi les modifications structurelles importantes de la Constitution. L'abolition de la chambre haute impliquerait une modification structurelle importante de la partie $\mathrm{V} \quad[\ldots]$ L'élimination du bicaméralisme rendrait inopérant ce mécanisme de contrôle et transformerait dans les faits la dynamique du processus de modification constitutionnelle. La structure constitutionnelle de la partie $\mathrm{V}$ serait fondamentalement modifiee dans son ensemble.

L'argument selon lequel l'abolition du Sénat n'entraînerait que des effets " accessoires " ou secondaires sur la partie V ne nous convainc pas non plus. Les effets de l'abolition du Sénat sur la partie V seraient directs et substantiels.

$[\ldots]$

La révision des modifications constitutionnelles par une chambre haute est un élément essentiel des procédures de modification prévues à la partie V. Le Sénat joue un rôle dans toutes ces procédures, exception faite de la procédure unilatérale provinciale. Le processus de modification constitutionnelle dans un régime unicaméral différerait, au

122. Id., par. 3 .

123. Id., par. 102.

124. Id., par. 106. 
plan qualitatif, du processus actuel. Il y aurait un acteur de moins dans le processus, et un mécanisme de contrôle disparaîtrait. Il faudrait décider si la procédure de modification peut être suivie en sa forme actuelle dans le cadre d'un régime unicaméral, ou si elle devrait être modifiée pour prévoir un nouveau mécanisme de contrôle exerçant la fonction autrefois assumée par la chambre haute. Ces questions se rapportent au fonctionnement de la formule de modification constitutionnelle et, à ce titre, selon l'al. 41e) de la Loi constitutionnelle de 1982, le consentement unanime du Parlement et des provinces est requis $^{125}$.

Les passages qui précèdent démontrent bien que la Cour suprême s'est refusée à appliquer, dans le cas de l'abolition du Sénat, la thèse formaliste qui avait été avancée par le procureur général du Canada. Au contraire, elle a tenu compte du rôle de la Chambre haute dans la structure constitutionnelle du pays et en est venue à la conclusion - à l'instar de la Cour d'appel du Québec avant elle126 - que l'abolition du Sénat changerait implicitement la procédure de modification constitutionnelle prévue dans la partie V de la Loi de 1982. Par conséquent, pareille abolition exigerait le consentement unanime des provinces (en plus de celui des autorités fédérales) en vertu de l'article 41e) de cette loi.

Somme toute, la Cour suprême a favorisé, dans le Renvoi relatif à la réforme du Sénat, une approche téléologique large en ce qui touche à l'interprétation des modalités de modification constitutionnelle établies par la partie V de la Loi de 1982127. Les

125. Id., par. 107,108 et 110 .

126. Projet de loi fédéral relatif au Sénat (Re), 2013 QCCA 1807 (CanLII).

127. Voir le Renvoi relatif à la réforme du Sénat, préc., note 16, par. 52, où la Cour suprême confirme ainsi l'application de l'approche téléologique large en matière de modification constitutionnelle :

À notre avis, l'argument selon lequel l'introduction d'élections consultatives n'est pas une modification de la Constitution est trop formaliste. En effet, il réduit la notion de modification constitutionnelle à la question de déterminer si le texte de la Constitution se trouve modifié. Cette approche restrictive ne respecte pas les exigences de l'interprétation et de la conception larges et téléologiques de la Constitution qui ont été 
juristes sauront ainsi que, désormais, ces dernières s'appliqueront non seulement aux changements apportés aux textes constitutionnels eux-mêmes, mais aussi aux transformations explicites ou implicites de la structure constitutionnelle propre au Canada.

\section{CONCLUSION}

Dans le présent texte, nous avons analysé l'approche qu'ont privilégiée les cours de justice jusqu'à présent en ce qui a trait à l'interprétation et à l'application des modalités de modification constitutionnelle de la partie V de la Loi de 1982.

Il ressort de notre analyse que les articles 44 et 45 de la Loi de 1982 - qui contiennent les pouvoirs unilatéraux de modification constitutionnelle - ne peuvent s'appliquer que pour les modifications qui ne touchent ni au compromis fédératif ni aux relations fédérales-provinciales (ou fédérales-provincialesterritoriales). Pour déterminer si la modification envisagée concerne ou non ces sujets, il faut tenir compte de son esprit tout autant que de son libellé.

Pour sa part, l'article 43 de la Loi de 1982 traite des modifications qui échappent à la portée de l'article 45 de cette loi, mais qui ne s'appliquent néanmoins qu'à une ou à quelques provinces, et non à toutes celles-ci. Lorsqu'il est mis en pratique, l'article 43 requiert alors le consentement de la Chambre des communes et du Sénat128 de même que celui de toutes les provinces directement et techniquement visées par la modification constitutionnelle en cause.

exposées précédemment. Certes, le libellé des dispositions concernant la nomination des sénateurs resterait le même, mais les nature et rôle fondamentaux du Sénat en tant que corps législatif complémentaire chargé de donner un second regard attentif aux projets de loi seraient considérablement modifiés.

128. Cela est dit sous réserve du fait que le Sénat ne dispose que d'un veto suspensif de 180 jours en vertu de l'article 47 de la Loi de 1982. 
Les autres modifications constitutionnelles - soit celles qui échappent à la portée des articles 43, 44 et 45 de la Loi de $1982-$ relèvent soit de l'un ou l'autre des articles 41 et 42 , selon le sujet sur lequel elles portent, soit de la procédure générale et résiduelle de l'article 38 (1) de la Loi de 1982.

Notons tout particulièrement que c'est la règle de l'unanimité, prévue par l'article 41 de la Loi de 1982, qui s'applique à la modification de la partie V de cette loi elle-même. Cela englobe non seulement les modifications techniques qui seraient apportées à la partie $\mathrm{V}$ en question, mais aussi les modifications influant de façon importante sur "la structure constitutionnelle canadienne ${ }^{129}$ ".

Comme on peut le voir, si l'interprétation téléologique prévaut en ce qui concerne les articles 44 et 45 , et même 41 , de la Loi de 1982, tel n'est cependant pas le cas en ce qui a trait à son article 43. Dans ce dernier cas, l'approche des tribunaux judiciaires se veut nettement plus technique, de sorte que seul le consentement de la province directement visée par la modification constitutionnelle souhaitée sera requis, en plus évidemment de celui des autorités fédérales.

Quant à l'expression "Constitution du Canada ", définie à l'article 52 (2) de la Loi de 1982, elle doit se voir donner une interprétation extensible. Elle contient assurément soit des textes qui n'y sont pas expressément mentionnés, soit des principes constitutionnels découlant de tels textes ${ }^{130}$.

129. Renvoi relatif à la réforme du Sénat, préc., note 16, par. 3 et 48 .

130. Dans le Renvoi relatif à la Loi sur la Cour suprême, préc., note 15, celle-ci n'a pas dit explicitement que la Loi sur la Cour suprême, préc., note 67, était enchâssée dans la Constitution canadienne. Elle a indiqué néanmoins que "[1]'alinéa 42(1)d) applique la procédure de modification 7/50 aux caractéristiques essentielles de la Cour, plutôt qu'à toutes les dispositions de la Loi sur la Cour suprême " (par. 94). La Cour suprême a également laissé entendre à différents endroits dans sa décision que l'article 4 (1) de même que les articles 5 et 6 de la Loi sur la Cour suprême étaient bel et bien enchâssés dans la Constitution du pays (par. 5, 19, 25, 49, 74, 91, 93, 103-105). Enfin, comme nous l'avons vu précédemment, la 
Grâce à des décisions récentes comme le Renvoi relatif à la Loi sur la Cour suprême ou le Renvoi relatif à la réforme du Sénat, le juriste est en mesure de mieux comprendre la dynamique propre à la partie V de la Loi de 1982. Celle-ci demeure toutefois éminemment complexe. Elle reste aussi relativement nébuleuse. Par exemple, il est loin d'être certain que l'article 43 de la Loi de 1982 permet d'ajouter des dispositions nouvelles à la Constitution du Canada plutôt que de simplement servir à la modification des dispositions existantes ${ }^{131}$.

Quoi qu'il en soit, on aurait tort de sous-estimer l'apport des tribunaux à la compréhension de la partie V de la Loi de 1982. Et davantage encore de croire que la modification constitutionnelle est, au Canada, un sujet statique, sans vie, sans intérêt. Au contraire, année après année, la jurisprudence canadienne a discuté de ce sujet; elle a cherché à le démystifier.

Loin d'être un ensemble normatif hermétique et terne, la Constitution canadienne est un arbre vivant, ainsi que le disait lord Sankey à l'occasion de l'affaire Edwards v. Attorney General for

Cour suprême a rejeté l'argument du procureur général du Canada voulant que la Loi sur la Cour suprême ne soit pas protégée constitutionnellement parce qu'elle n'est pas mentionnée à l'article 52 de la Loi de 1982.

131. Guy TRemblay, "La portée élargie de la procédure bilatérale de modification de la Constitution du Canada ", (2011) 41,2 R.G.D. 417, 448, a examiné l'article 43 de la Loi de 1982. Il en est venu à la conclusion que cet article permettait possiblement d'ajouter dans la Constitution des dispositions nouvelles, tout en autorisant évidemment la modification des dispositions existantes :

Malgré la rigidité de ses dispositions principales, rigidité que des lois du fédéral et de certaines provinces ont accrue, la procédure de modification de la Constitution du Canada recèle un mécanisme qui permettrait au Québec d'obtenir satisfaction par simple entente avec le fédéral. Il se trouve dans l'article 43 de la Loi constitutionnelle de 1982, lequel permet, selon nous, d'ajouter des dispositions nouvelles dans la Constitution et permet au surplus de greffer des garanties linguistiques et culturelles aux dispositions existantes qui octroient déjà un certain statut particulier au Québec. 
Canada $^{132}$. Et si les racines de cet arbre puisent profondément et finement dans l'histoire, ses ramifications, quant à elles, se révèlent bien imposantes et actuelles.

132. Edwards v. Attorney General for Canada, [1930] A.C. 124, p. 136. 